\title{
Efficient Lattice Method for Valuing of Options with Barrier in a Regime Switching Model
}

\author{
Youngchul Han ${ }^{1}$ and Geonwoo Kim² \\ ${ }^{1}$ Department of Mathematics, Yonsei University, Seoul 120-749, Republic of Korea \\ ${ }^{2}$ Department of Mathematical Science, Seoul National University, Seoul 151-747, Republic of Korea \\ Correspondence should be addressed to Geonwoo Kim; geonwoo@yonsei.ac.kr
}

Received 25 July 2016; Revised 7 September 2016; Accepted 14 September 2016

Academic Editor: Francisco R. Villatoro

Copyright (c) 2016 Y. Han and G. Kim. This is an open access article distributed under the Creative Commons Attribution License, which permits unrestricted use, distribution, and reproduction in any medium, provided the original work is properly cited.

\begin{abstract}
We propose an efficient lattice method for valuation of options with barrier in a regime switching model. Specifically, we extend the trinomial tree method of Yuen and Yang (2010) by calculating the local average of prices near a node of the lattice. The proposed method reduces oscillations of the lattice method for pricing barrier options and improves the convergence speed. Finally, computational results for the valuation of options with barrier show that the proposed method with interpolation is more efficient than the other tree methods.
\end{abstract}

\section{Introduction}

Barrier options are most popular options among the exotic options. The barrier options are the contingent claims whose payoffs depend on the relationship between the specified barriers and the path of the underlying asset. If the underlying asset crosses a specified barrier or barriers before the maturity, a barrier option of a knock-out type becomes worthless. And a barrier option of a knock-in type is activated when the underlying asset crosses a specified barrier. Barrier options are important in the financial market because barrier options are cheaper than standard European options and provide flexibility. Thus, they have been studied by many researchers.

The pricing formula of a down-and-out barrier option is first presented by Merton [1] under the Black-Scholes model. Rubinstein and Reiner [2] proposed pricing formulas of various kinds of options with single barrier. For the pricing of double barrier options, Kunitomo and Ikeda [3] proposed a pricing formula of options with exponentially curved barriers. The results are expressed as an infinite sum of normal distribution functions. Alternatively, Geman and Yor [4] provided the Laplace transform of the double barrier option price by a probabilistic approach. Other researchers also presented the double barrier option price formula using different methods including the path counting and the method of images (for the details, see Sidenius [5], Lin [6], and Buchen and Konstandatos [7]).

The various types of barrier option are developed by many researchers. Most studies are carried out using the BlackScholes model. However, it is well known that the BlackScholes model is not adequate to explain the market behavior of option prices. In other words, the Black-Scholes model does not explain well the volatility smile phenomenon in the real market. For the more realistic model, many extensions to the Black-Scholes model have been introduced for valuing options. Among many extensions, we focus on the regime switching model for valuing options with barrier in this paper.

The regime switching model is one of the popular alternative models to overcome the limitations of Black-Sholes model. Since the regime switching model was first introduced by Hamilton [8], there have been many studies for the regime switching model in finance area. In particular, many researchers have used the regime switching model which leads to transition of volatilities of the underlying assets for valuation of various options. Naik [9] derived the price of the European option using the conditional probability density functions of occupation time of the volatility in high state. Buffington and Elliott [10] provided a method for valuation of the American options by partial differential equation 
approach. Guo and Zhang [11] derived a closed-form pricing solution for the perpetual American options. In addition, Boyle and Draviam [12] presented the general numerical methods for valuation of exotic options. Elliott et al. [13] also dealt with pricing of barrier options with regime switching.

The lattice methods for valuation of options with regime switching have received much attention by many researchers in recent year. Bollen [14] first introduced a lattice method for valuation of options with a single underlying asset in regime switching model. Lin [6] suggested the new recombining tree method for efficient valuation of the European and the American options with regime switching. Liu and Zhao [15] extended the method of Lin [6] to options with two underlying assets which follow the regime switching model. In addition, Yuen and Yang [16] developed a trinomial tree method for valuation of options in a regime switching model. Costabile et al. [17] presented a multinomial approach for pricing options under the regime switching jump-diffusion model. Costabile [18] proposed a trinomial lattice model for approximating the evolution of the investment fund value with regime switching.

In this paper, we propose the efficient lattice methods for pricing options including American type options in a regime switching model. More concretely, we develop the trinomial tree methods for valuing options with barriers. In order to construct these lattice methods, we adopt the local average method and the interpolation method. As expected, we can find that our lattice methods provide efficiently the prices of options with barriers.

The remainder of the paper is organized as follows. In Section 2, we review the trinomial method for option pricing in a regime switching model. In Section 3, we propose efficient lattice method based on the local averages and interpolation. Section 4 presents the numerical results of diverse options with barrier. Finally, we give the concluding remarks in Section 5.

\section{Trinomial Tree Method for the Regime Switching Model}

In this section, we describe the tree method for the regime switching model to price the options. For this, we review the trinomial tree method of Yuen and Yang [16].

In order to describe the evolution of $k$-state of the economy with regime switching, we first define that $X(t)$ is a continuous-time Markov chain with finite $k$-state space $\chi:=$ $\left(x_{1}, x_{2}, \ldots, x_{k}\right)$, which represents general market trends and economic conditions. And we assume that $X(t)$ is observable. Then, under the risk neutral measure, the dynamics are the underlying asset with regime switching are

$$
d S(t)=r(X(t)) S(t) d t+\sigma(X(t)) S(t) d W(t)
$$

where $W(t)$ is a standard Brownian motion. For observed state $k$ at time $t$, the interest rate $r\left(x_{k}\right)=r_{k}$ and the volatility $\sigma\left(x_{k}\right)=\sigma_{k}$ are constants. In addition, we assume that $A(t)=\left[a_{i j}(t)\right]_{i, j=1, \ldots, k}$ is the generator matrix of $X(t)$ to be state dependent. Then functions of elements are continuous and bounded and are constants satisfying $a_{i j}(t) \geq 0$ for $i \neq j$ and $\sum_{j} a_{i j}(t)=0$ for each $i=1, \ldots, k$.

We now introduce the tree method of Yuen and Yang for valuing options with regime switching. Let $\pi_{u}, \pi_{m}, \pi_{d}$ be the risk neutral probabilities of the underlying asset price up, middle, and down, respectively. We put $N+1$ uniform time grids between 0 and maturity $T$ with time mesh $\Delta t=T / N$. Then we have

$$
\begin{aligned}
\pi_{u} e^{\lambda \sigma \sqrt{\Delta t}}+\pi_{m}+\pi_{d} e^{-\lambda \sigma \sqrt{\Delta t}} & =e^{r \Delta t} \\
\left(\pi_{u}+\pi_{d}\right) \lambda^{2} \sigma^{2} \Delta t & =\sigma^{2} \Delta t
\end{aligned}
$$

where $r$ is the risk free interest rate and $\lambda$ should be greater than 1 , so that the risk neutral probability measure exists.

We choose $e^{ \pm \sigma \sqrt{\Delta t}}$ as the size of the up and down move, where $\sigma>\max _{i \in\{1, \ldots, k\}} \sigma_{i}$. For each $X(t)=x_{i},(i=1, \ldots, k)$, let $\pi_{u}^{i}, \pi_{m}^{i}, \pi_{d}^{i}$ be the risk neutral probabilities of the underlying asset price up, middle, and down, respectively. Then, for the implementation of the regime switching model, we can obtain the following equations:

$$
\begin{aligned}
\pi_{u}^{i} e^{\sigma \sqrt{\Delta t}}+\pi_{m}^{i}+\pi_{d}^{i} e^{-\sigma \sqrt{\Delta t}} & =e^{r_{i} \Delta t} \\
\left(\pi_{u}^{i}+\pi_{d}^{i}\right) \sigma^{2} \Delta t & =\sigma_{i}^{2} \Delta t .
\end{aligned}
$$

If $\lambda_{i}$ is defined as $\sigma / \sigma_{i}$ for each $i$, we have $\lambda_{i}>1$ and $\pi_{u}^{i}, \pi_{m}^{i}, \pi_{d}^{i}$ can be expressed in terms of $\lambda_{i}$ as

$$
\begin{aligned}
& \pi_{m}^{i}=1-\frac{\sigma_{i}^{2}}{\sigma^{2}}=1-\frac{1}{\lambda_{i}^{2}}, \\
& \pi_{u}^{i}=\frac{e^{r_{i} \Delta t}-e^{-\sigma \sqrt{\Delta t}}-\left(1-1 / \lambda_{i}^{2}\right)\left(1-e^{-\sigma \sqrt{\Delta t}}\right)}{e^{\sigma \sqrt{\Delta t}}-e^{-\sigma \sqrt{\Delta t}}}, \\
& \pi_{d}^{i}=\frac{-e^{r_{i} \Delta t}+e^{\sigma \sqrt{\Delta t}}-\left(1-1 / \lambda_{i}^{2}\right)\left(e^{\sigma \sqrt{\Delta t}}-1\right)}{e^{\sigma \sqrt{\Delta t}}-e^{-\sigma \sqrt{\Delta t}}} .
\end{aligned}
$$

Here, by the suggestion of Yuen and Yang [16], we choose $\sigma$ as

$$
\sigma=\max _{i \in\{1, \ldots, k\}} \sigma_{i}+(\sqrt{1.5}-1) \bar{\sigma}
$$

where $\bar{\sigma}$ is the mean of $\sigma_{i}$.

Let $V_{t, n}^{i}$ be the option value with strike $K$ at node $n$ and time step $t$ when the state at time step $t$ is $i$. Then the option values at each node can be calculated by backward induction algorithm with the terminal condition $V_{N, n}^{i}=\left(S_{N, n}-K\right)^{+}$ (call option) or $V_{N, n}^{i}=\left(K-S_{N, n}\right)^{+}$(put option) for all $i$, where underlying stock price $S_{N, n}$ is given by $S_{N, n}=$ $S_{0} e^{(n-1-N) \sigma \sqrt{\Delta t}}$. Since the Markov chain is independent of the Brownian motions, the transition probabilities are not affected by changing the probability measure. Therefore, the 
option values under the regime switching model can be calculated by employing the following equation recursively:

$$
\begin{aligned}
V_{t, n}^{i} & =e^{-r_{i} \Delta t}\left[\sum _ { j = 1 } ^ { k } p _ { i j } \left(\pi_{u}^{i} V_{t+1, n+2}^{j}+\pi_{m}^{i} V_{t+1, n+1}^{j}\right.\right. \\
& \left.\left.+\pi_{d}^{i} V_{t+1, n}^{j}\right)\right]
\end{aligned}
$$

where $p_{i j}$ is given by

$$
e^{A \Delta t}=I+\sum_{l=1}^{\infty} \frac{(\Delta t)^{l} A^{l}}{l !}=\left(\begin{array}{ccc}
p_{11} & \cdots & p_{1 k} \\
\vdots & \ddots & \vdots \\
p_{k 1} & \cdots & p_{k k}
\end{array}\right),
$$

with the identity matrix $I$ and the generator matrix $A$.

\section{Numerical Methods}

In this section, we propose the efficient lattice methods for pricing options with barrier including American type options. We construct the trinomial tree method using local averages with regime switching (LARS) in Section 3.1. Finally, we propose the LARSI method by combining LARS method and interpolation method in Section 3.2.

3.1. LARS Method. The tree method using local averages was introduced by Moon and Kim [19]. They modified the standard binomial tree method based on local averages of option prices and showed that their method is more effective than other methods computationally. By combining the local average method into the trinomial tree method mentioned in the previous section, we propose the trinomial tree method for the efficient valuation of options with regime switching.

Let $Y(t)=\ln (S(t))$. Then, when the state at time 0 is $i$, the option value can be computed by

$$
V^{i}:=V_{0}^{i}(y)=e^{-r_{i} T} E[\Lambda(Y(T)) \mid Y(0)=y],
$$

where $\Lambda(\cdot)$ is a payoff function at maturity $T$.
We denote that the value of underlying asset is $Y_{N, j}=$ $Y(0)+h j$ for $j=0, \ldots, 2 N+1$, where $h=\sigma \sqrt{\Delta t}$, and divide the interval $[Y(0)-(1 / 2) h, Y(0)+2 N+(3 / 2) h]$ at the maturity into $2 N+1$ nonoverlapping intervals of the uniform length $h$. Then the average option prices on each interval can be calculated by

$$
\bar{V}_{N, j}=\frac{1}{h} \int_{Y_{N, j-0.5}}^{Y_{N, j+0.5}} \Lambda(s) d s, \quad j=0, \ldots, 2 N+1,
$$

where $Y_{N, j}=Y(0)+h j$ at the maturity.

We consider the average option prices at time $t$ with regime $i$. Then the average option prices satisfy the following relation:

$$
\begin{aligned}
\bar{V}_{t, j}^{i} & =e^{-r_{i} \Delta t}\left[\sum _ { m = 1 } ^ { k } p _ { i m } \left(\pi_{u}^{i} \bar{V}_{t+1, j+2}^{m}+\pi_{m}^{i} \bar{V}_{t+1, j+1}^{m}\right.\right. \\
& \left.\left.+\pi_{d}^{i} \bar{V}_{t+1, j}^{m}\right)\right]
\end{aligned}
$$

for $j=0, \ldots, 2 n+1$.

From relation (10), we can obtain the option price $\bar{V}_{0,0}^{i}$ at time $t=0$ with regime $i$. We further use the following scheme to reduce the approximation error of the option price (for more details, see the Appendix)

$$
V^{i}:=V_{0,0}^{i}=-\frac{1}{24} \bar{V}_{0,-1}^{i}+\frac{13}{12} \bar{V}_{0,0}^{i}-\frac{1}{24} \bar{V}_{0,1}^{i} .
$$

Then $V_{0,0}^{i}$ finally becomes the standard option price with regime $i$ by the LARS method.

For the American option, which allows early exercise of the option before the maturity, we find the optimal boundary $S^{*}$ at each regime $i$ by

$$
\max \left\{V_{t}^{i}(y), \Lambda_{t}(y)\right\}= \begin{cases}V_{t}^{i}(y), & \text { if } S^{*} \leq y \\ \Lambda_{t}(y), & \text { if } S^{*} \geq y\end{cases}
$$

where $V_{t}^{i}(y)$ is the option price with regime $i$ from the backward process of the lattice at time $t$ and $\Lambda_{t}(y)$ is the exercise price at time $t$. Then we have

$$
\int_{Y_{n, j}-h / 2}^{Y_{n, j}+h / 2} \max \left\{V_{t}^{i}(s), \Lambda_{t}(s)\right\} d s= \begin{cases}\max \left\{\int_{Y_{n, j}-h / 2}^{Y_{n, j}+h / 2} V_{t}^{i}(s) d s, \int_{Y_{n, j}-h / 2}^{Y_{n, j}+h / 2} \Lambda_{t}(s) d s\right\}, & \text { if } S^{*} \leq Y_{n, j}-\frac{h}{2} \text { or } S^{*} \geq Y_{n, j}+\frac{h}{2} \\ \int_{Y_{n, j}-h / 2}^{s^{*}} \Lambda_{t}(s) d s+\int_{S^{*}}^{Y_{n, j}+h / 2} V_{t}^{i}(s) d s, & \text { if } Y_{n, j}-\frac{h}{2}<S^{*}<Y_{n, j}+\frac{h}{2}\end{cases}
$$

If $S^{*}$ is in the interval $\left[Y_{n, j}-2 / h, Y_{n, j}+2 / h\right]$, the error between $\max \left\{\int_{Y_{n, j}-h / 2}^{Y_{n, j}+h / 2} V_{t}^{i}(s) d s, \int_{Y_{n, j}-h / 2}^{Y_{n, j}+h / 2} \Lambda_{t}(s) d s\right\}$ and
$\int_{Y_{n, j}-h / 2}^{S^{*}} \Lambda_{t}(s) d s+\int_{S^{*}}^{Y_{n, j}+h / 2} V_{t}^{i}(s) d s$ gets smaller when $h$ goes to 0 . Let $\bar{V}_{n, j}=(1 / h) \int_{Y_{n, j}-h / 2}^{Y_{n, j}+h / 2} \Lambda_{t}(s) d s$. Then we can update 
the option price at node $n$ with regime $i$ by $\max \left\{\bar{V}_{n, j}^{i}, \bar{\Lambda}_{n, j}\right\}$, and the American option price with regime $i$ is computed by applying the backward iteration (10).

3.2. LARSI Method. When the lattice methods such as binomial or trinomial trees are used for valuing of the barrier options, it is well known that a large number of time steps are required to obtain reasonably accurate results. Therefore, the convergence speed of the lattice methods becomes very slow. This phenomenon occurs since barrier being assumed by the lattice is different from the true barrier. In order to overcome this phenomenon, the interpolation method has been used when options with barrier are priced by the lattice methods. We propose the LARSI method by combining the interpolation method into LARS method, which provides efficiently accurate prices of options with barrier in a regime switching model.

To describe the LARSI method, we define the inner barrier as the barrier formed by the nodes just on the inside of the true barrier and the outer barrier as the barrier formed by nodes just outside the true barrier. Then the LARSI method is as follows.

LARSI Method. The LARSI method is as follows:

(1) Compute the price $V_{B_{1}}^{i}$ of the barrier option with regime $i$ by LARS method on the assumption that the inner barrier $B_{1}$ is the true barrier $B$.

(2) Compute the price $V_{B_{2}}^{i}$ of the barrier option with regime $i$ by LARS method on the assumption that the outer barrier $B_{2}$ is the true barrier $B$.

(3) Compute the price of the barrier option with regime $i$ as

$$
V^{i}=V_{B_{1}}^{i}+\left(B-B_{1}\right) \frac{V_{B_{2}}^{i}-V_{B_{1}}^{i}}{B_{2}-B_{1}} .
$$

\section{Numerical Results}

Based on the model described in the previous section, we calculate the prices of various options with regime. In this section, we study all types of barrier options including the European type and the American type. Specifically, prices of these options with two-state regime (it was shown in [20] that two-state regime switching model is sufficient for accurate option pricing in the real market. Also, it is possible to extend multistate regime switching model easily) are calculated using the LARS and LARSI (LARS-type) methods, and the accuracy and efficiency of LARS-type method for valuing options with barrier are shown by comparing with the results given in $[12,16]$.

First, we assume that the initial underlying asset price $S_{0}$ and the strike price are set to be 100 . The volatility of the underlying asset in regime 1 and regime 2 is 0.15 and 0.25 , respectively. The option has 1-year maturity, and the trinomial tree is set to have 1000 time steps. The generators of the regime switching process of LARS-type are

$$
\begin{gathered}
\left(\begin{array}{cc}
-0.5 & 0.5 \\
0.5 & -0.5
\end{array}\right), \\
\left(\begin{array}{cc}
-1 & 1 \\
1 & -1
\end{array}\right),
\end{gathered}
$$

for the above parameter sets, respectively. We present the numerical results for European call option prices, and the results are compared with benchmarks Naik (Naik) [9], Boyle and Draviam (B\&D) [12], and Yuen and Yang (Y\&Y) [16] in Table 1. Table 1 shows that numerical results for the option prices obtained by using the LARS method are very close to the value obtained by the analytical solutions derived in [9] and also close to those obtained using partial differential equation in [12]. This verifies that the LARS-type method is applicable (cf. [16]).

We now study the values of diverse options by the LARS method. The underlying asset is assumed to be a stock with the initial price 100, following the geometric Brownian motion with no dividend and two-state regime. In regime 1 , the risk free interest rate is $4 \%$ and the volatility of stock is 0.25 ; in regime 2 , the risk free interest rate is $6 \%$ and the volatility of stock is 0.35 . All options expire in one year with a strike price 100 . The generator for the regime switching model of LARS-type is taken to be

$$
\left(\begin{array}{cc}
-0.5 & 0.5 \\
0.5 & -0.5
\end{array}\right)
$$

The transition probabilities of the offshoot of state up, middle, and down with 20 time steps are $0.1817,0.6413$, and 0.1770 in regime 1 and $0.3512,0.2970$, and 0.3518 in regime 2 , respectively. These values depend on the size of time step, but the values with other sizes of times step are not much different from these values because the time step is small in general. We carry out the experiments to see significant properties of the proposed method.

Table 2 shows that the prices of the European call and the European put options converge rapidly. " $N$ " is a number of time steps used in the calculation, "Difference" refers to a difference in price calculated using various numbers of time steps, and "Ratio" is a ratio of the difference. We know that the price of the derivative using the Cox-Ross-Rubinstein (CRR) model converges to the corresponding price under the simple geometric Brownian motion model and that the speed of convergence can have order 1; that is, the error of the price is halved if the number of time steps is doubled (for more details, see $[21,22])$. We can see that most of the ratios shown in the tables are close to 0.5 . The ratios of the European call option for regime 2 are also close to 0.5 . Therefore, the convergence patterns in regime 1 and regime 2 are more stable than the method of Y\&Y. If we apply put-call parity to each of the regimes, the interest rate implied in two regimes is $4.367 \%$ and $5.631 \%$, respectively, in the 5120 time steps case. This is reasonable because both of them are between $4 \%$ and $6 \%$, and the interest rate implied by the numerical results 
TABLE 1: Comparison of different methods in pricing the European call option in LARS method.

\begin{tabular}{|c|c|c|c|c|c|c|c|c|}
\hline \multirow{2}{*}{$S_{0}$} & \multicolumn{4}{|c|}{ Regime 1} & \multicolumn{4}{|c|}{ Regime 2} \\
\hline & Naik & $\mathrm{B} \& \mathrm{D}$ & $\mathrm{Y} \& \mathrm{Y}$ & LARS & Naik & $\mathrm{B} \& \mathrm{D}$ & $Y \& Y$ & LARS \\
\hline \multicolumn{9}{|c|}{ European call option 1} \\
\hline 94 & 5.8620 & 5.8579 & 5.8615 & 5.8612 & 8.2292 & 8.2193 & 8.2297 & 8.2296 \\
\hline 96 & 6.9235 & 6.9178 & 6.9229 & 6.9226 & 9.3175 & 9.3056 & 9.3181 & 9.3180 \\
\hline 98 & 8.0844 & 8.0775 & 8.0827 & 8.0834 & 10.4775 & 10.4647 & 10.4772 & 10.4779 \\
\hline 100 & 9.3401 & 9.3324 & 9.3369 & 9.3390 & 11.7063 & 11.6929 & 11.7049 & 11.7066 \\
\hline 102 & 10.6850 & 10.6769 & 10.6828 & 10.6839 & 13.0008 & 12.9870 & 13.0001 & 13.0010 \\
\hline 104 & 12.1127 & 12.1045 & 12.1108 & 12.1114 & 14.3575 & 14.3436 & 14.3571 & 14.3576 \\
\hline 106 & 13.6161 & 13.6082 & 13.6143 & 13.6147 & 15.7729 & 15.7591 & 15.7725 & 15.7729 \\
\hline \multicolumn{9}{|c|}{ European call option 2} \\
\hline 94 & 6.2748 & 6.2705 & 6.2760 & 6.2758 & 7.8905 & 7.8844 & 7.8943 & 7.8942 \\
\hline 96 & 7.3408 & 7.3352 & 7.3422 & 7.3420 & 8.9747 & 8.9680 & 8.9789 & 8.9788 \\
\hline 98 & 8.5001 & 8.4938 & 8.5010 & 8.5017 & 10.1335 & 10.1264 & 10.1374 & 10.1380 \\
\hline 100 & 9.7489 & 9.7423 & 9.7489 & 9.7509 & 11.3641 & 11.3568 & 11.3673 & 11.3690 \\
\hline 102 & 11.0820 & 11.0755 & 11.0833 & 11.0844 & 12.6631 & 12.6659 & 12.6674 & 12.6683 \\
\hline 104 & 12.4937 & 12.4877 & 12.4959 & 12.4965 & 14.0267 & 14.0197 & 14.0317 & 14.0322 \\
\hline 106 & 13.9777 & 13.9726 & 13.9805 & 13.9810 & 15.4510 & 15.4446 & 15.4565 & 15.4569 \\
\hline
\end{tabular}

TABLE 2: Pricing the European standard option with the LARS method.

\begin{tabular}{|c|c|c|c|c|c|c|c|c|c|c|c|c|}
\hline \multirow{3}{*}{$N$} & \multicolumn{6}{|c|}{ European standard call option } & \multicolumn{6}{|c|}{ European standard put option } \\
\hline & \multicolumn{3}{|c|}{ Regime 1} & \multicolumn{3}{|c|}{ Regime 2} & \multicolumn{3}{|c|}{ Regime 1} & \multicolumn{3}{|c|}{ Regime 2} \\
\hline & Price & Difference & Ratio & Price & Difference & Ratio & Price & Difference & Ratio & Price & Difference & Ratio \\
\hline 20 & 12.7776 & -0.0094 & 0.5159 & 15.8842 & -0.0597 & 0.4971 & 8.4479 & 0.0192 & 0.4939 & 10.3216 & -0.0158 & 0.4899 \\
\hline 40 & 12.7681 & -0.0049 & 0.5072 & 15.8245 & -0.0297 & 0.4985 & 8.4671 & 0.0095 & 0.4972 & 10.3058 & -0.0077 & 0.4948 \\
\hline 80 & 12.7633 & -0.0025 & 0.5034 & 15.7948 & -0.0148 & 0.4993 & 8.4766 & 0.0047 & 0.4987 & 10.2981 & -0.0038 & 0.4974 \\
\hline 160 & 12.7608 & -0.0012 & 0.5017 & 15.7801 & -0.0074 & 0.4996 & 8.4814 & 0.0024 & 0.4993 & 10.2942 & -0.0019 & 0.4987 \\
\hline 320 & 12.7596 & -0.0006 & 0.5008 & 15.7727 & -0.0037 & 0.4998 & 8.4837 & 0.0012 & 0.4997 & 10.2923 & -0.0010 & 0.4993 \\
\hline 640 & 12.7589 & -0.0003 & 0.5004 & 15.7690 & -0.0018 & 0.4999 & 8.4849 & 0.0006 & 0.4999 & 10.2914 & -0.0005 & 0.4997 \\
\hline 1280 & 12.7586 & -0.0002 & 0.5001 & 15.7671 & -0.0009 & 0.4999 & 8.4855 & 0.0003 & 0.5000 & 10.2909 & -0.0002 & 0.4998 \\
\hline 2560 & 12.7585 & -0.0001 & & 15.7662 & -0.0004 & & 8.4858 & 0.0001 & & 10.2907 & -0.0001 & \\
\hline 5120 & 12.7584 & & & 15.7658 & & & 8.4859 & & & 10.2906 & & \\
\hline
\end{tabular}

in regime 1 is closer to the rate in regime 1 , while the same happens for regime 2. Interestingly, the deviations between the current interest rate and the interest rate implied by the put-call parity in both regimes are close to $0.37 \%$. This is because of the symmetry of the two regimes in terms of the transition probabilities.

The result of the American option is similar to that of the CRR model (Table 3). The prices of the American call option found by the LARS method are the same as the European call option. It is consistent with the understanding that the American call option is always not optimal to be exercised before expiration if there is no dividend being distributed. The prices of the American put option in the table are larger than those of the European put option, meaning that early exercise of the option is sometimes preferred and there may be some situations when we have to exercise the American put option before expiration. The convergence pattern of the American put option is similar to the European one. In the method of Y\&Y, the convergence pattern of the American put option with regime 2 is unstable even if it is fast, whereas the LARS method gives stable convergence patterns.

For the out-type barrier (down-and-out and up-and-out barrier) options, the prices found in both regimes are smaller than those of the European call option due to the presence of the barriers. The barrier level is set as 90 for the downand-out barrier options and 110 for the up-and-out barrier options. The prices of down-and-out barrier option in the two regimes are closer to each other compared with those of the European option (Tables 4-7). Although the volatility of regime 2 is greater and has a higher chance to achieve a higher option value at expiration, the high volatility also increases the chance of hitting the barriers and thus eliminates its advantage. The convergence pattern of the down-and-out barrier option is very complicated. It might be the effect of quadratic approximation errors in pricing barrier options. It is difficult to get any conclusions from the numerical results. However, we can see that the prices of out-type options are oscillating and the differences still have a decreasing trend 
TABle 3: Pricing the American standard option with the LARS method.

\begin{tabular}{|c|c|c|c|c|c|c|c|c|c|c|c|c|}
\hline \multirow{3}{*}{$N$} & \multicolumn{6}{|c|}{ American standard call option } & \multicolumn{6}{|c|}{ American standard put option } \\
\hline & \multicolumn{3}{|c|}{ Regime 1} & \multicolumn{3}{|c|}{ Regime 2} & \multicolumn{3}{|c|}{ Regime 1} & \multicolumn{3}{|c|}{ Regime 2} \\
\hline & Price & Difference & Ratio & Price & Difference & Ratio & Price & Difference & Ratio & Price & Difference & Ratio \\
\hline 20 & 12.7776 & -0.0094 & 0.5159 & 15.8842 & -0.0597 & 0.4971 & 8.8606 & 0.0236 & 0.5507 & 10.9320 & -0.0165 & 0.4796 \\
\hline 40 & 12.7681 & -0.0049 & 0.5072 & 15.8245 & -0.0297 & 0.4985 & 8.8842 & 0.0130 & 0.4353 & 10.9154 & -0.0079 & 0.6365 \\
\hline 80 & 12.7633 & -0.0025 & 0.5034 & 15.7948 & -0.0148 & 0.4993 & 8.8972 & 0.0057 & 0.4692 & 10.9075 & -0.0050 & 0.5010 \\
\hline 160 & 12.7608 & -0.0012 & 0.5017 & 15.7801 & -0.0074 & 0.4996 & 8.9028 & 0.0027 & 0.4577 & 10.9024 & -0.0025 & 0.5487 \\
\hline 320 & 12.7596 & -0.0006 & 0.5008 & 15.7727 & -0.0037 & 0.4998 & 8.9055 & 0.0012 & 0.4671 & 10.8999 & -0.0014 & 0.5362 \\
\hline 640 & 12.7589 & -0.0003 & 0.5004 & 15.7690 & -0.0018 & 0.4999 & 8.9067 & 0.0006 & 0.4812 & 10.8985 & -0.0007 & 0.5156 \\
\hline 1280 & 12.7586 & -0.0002 & 0.5001 & 15.7671 & -0.0009 & 0.4999 & 8.9073 & 0.0003 & 0.4756 & 10.8978 & -0.0004 & 0.5194 \\
\hline 2560 & 12.7585 & -0.0001 & & 15.7662 & -0.0004 & & 8.9075 & 0.0002 & & 10.8974 & -0.0002 & \\
\hline 5120 & 12.7584 & & & 15.7658 & & & 8.9077 & & & 10.8972 & & \\
\hline
\end{tabular}

TABLE 4: Pricing the European down-and-out barrier call option with the LARS-type method.

\begin{tabular}{|c|c|c|c|c|c|c|c|c|c|c|c|c|}
\hline \multirow{3}{*}{$N$} & \multicolumn{6}{|c|}{ LARS method } & \multicolumn{6}{|c|}{ LARSI method } \\
\hline & \multicolumn{3}{|c|}{ Regime 1} & \multicolumn{3}{|c|}{ Regime 2} & \multicolumn{3}{|c|}{ Regime 1} & \multicolumn{3}{|c|}{ Regime 2} \\
\hline & Price & Difference & Ratio & Price & Difference & Ratio & Price & Difference & Ratio & Price & Difference & Ratio \\
\hline 20 & 11.6335 & -1.4914 & -0.1840 & 13.4120 & -2.1980 & -0.1660 & 8.7988 & -0.0745 & -2.1090 & 9.5653 & -0.0680 & -1.8901 \\
\hline 40 & 10.1420 & 0.2745 & -1.0490 & 11.2140 & 0.3648 & -1.0776 & 8.7243 & 0.1571 & 0.3370 & 9.4974 & 0.1285 & 0.3463 \\
\hline 80 & 10.4165 & -0.2879 & 2.1678 & 11.5788 & -0.3931 & 2.0738 & 8.8814 & 0.0529 & 0.0803 & 9.6258 & 0.0445 & 0.0582 \\
\hline 160 & 10.1286 & -0.6242 & 0.0853 & 11.1857 & -0.8152 & 0.0839 & 8.9343 & 0.0042 & 3.8905 & 9.6703 & 0.0026 & 5.3210 \\
\hline 320 & 9.5043 & -0.0533 & -0.9695 & 10.3705 & -0.0684 & -0.9553 & 8.9386 & 0.0165 & 0.8524 & 9.6729 & 0.0138 & 0.8811 \\
\hline 640 & 9.4511 & 0.0516 & -8.5059 & 10.3021 & 0.0654 & -8.4494 & 8.9551 & 0.0141 & -0.1603 & 9.6867 & 0.0121 & -0.1824 \\
\hline 1280 & 9.5027 & -0.4392 & -0.3926 & 10.3674 & -0.5523 & -0.3892 & 8.9692 & -0.0023 & -1.0424 & 9.6988 & -0.0022 & -0.8923 \\
\hline 2560 & 9.0635 & 0.1724 & & 9.8151 & 0.2150 & & 8.9669 & 0.0024 & & 9.6966 & 0.0020 & \\
\hline 5120 & 9.2359 & & & 10.0301 & & & 8.9693 & & & 9.6986 & & \\
\hline
\end{tabular}

TABLE 5: Pricing the European down-and-out barrier put option with the LARS-type method.

\begin{tabular}{|c|c|c|c|c|c|c|c|c|c|c|c|c|}
\hline \multirow{3}{*}{$N$} & \multicolumn{6}{|c|}{ LARS method } & \multicolumn{6}{|c|}{ LARSI method } \\
\hline & \multicolumn{3}{|c|}{ Regime 1} & \multicolumn{3}{|c|}{ Regime 2} & \multicolumn{3}{|c|}{ Regime 1} & \multicolumn{3}{|c|}{ Regime 2} \\
\hline & Price & Difference & Ratio & Price & Difference & Ratio & Price & Difference & Ratio & Price & Difference & Ratio \\
\hline 20 & 0.5299 & -0.3710 & -0.1251 & 0.2961 & -0.2107 & -0.1278 & 0.1001 & -0.0005 & 26.3194 & 0.0545 & -0.0011 & 6.1249 \\
\hline 40 & 0.1589 & 0.0464 & -0.7938 & 0.0854 & 0.0269 & -0.7560 & 0.0996 & -0.0134 & 0.5454 & 0.0534 & -0.0070 & 0.5881 \\
\hline 80 & 0.2053 & -0.0369 & 1.6459 & 0.1123 & -0.0204 & 1.6562 & 0.0861 & -0.0073 & 0.0927 & 0.0465 & -0.0041 & 0.0881 \\
\hline 160 & 0.1684 & -0.0607 & 0.0557 & 0.0919 & -0.0337 & 0.0534 & 0.0788 & -0.0007 & 2.7990 & 0.0424 & -0.0004 & 2.8866 \\
\hline 320 & 0.1078 & -0.0034 & -1.2756 & 0.0582 & -0.0018 & -1.3459 & 0.0782 & -0.0019 & 0.9838 & 0.0420 & -0.0010 & 1.0095 \\
\hline 640 & 0.1044 & 0.0043 & -6.7772 & 0.0564 & 0.0024 & -6.6444 & 0.0763 & -0.0019 & -0.2083 & 0.0410 & -0.0011 & -0.2195 \\
\hline 1280 & 0.1087 & -0.0292 & -0.3595 & 0.0588 & -0.0161 & -0.3591 & 0.0744 & 0.0004 & -0.7057 & 0.0399 & 0.0002 & -0.6565 \\
\hline 2560 & 0.0795 & 0.0105 & & 0.0427 & 0.0058 & & 0.0748 & -0.0003 & & 0.0401 & -0.0001 & \\
\hline 5120 & 0.0900 & & & 0.0485 & & & 0.0745 & & & 0.0400 & & \\
\hline
\end{tabular}

in absolute value apart from converging uniformly in one direction. For the up-and-out barrier option, the prices of the option are oscillating and do not converge uniformly (Tables $8-11)$. Therefore, it is hard to conclude the efficiency of the LARS method for calculating up-and-out barrier option.

For further improvement of the convergence speed and stability of LARS method in valuing options with barrier, we apply the LARSI method. In down-and-out barrier option case, the convergence patterns of LARSI method are more stable and faster than LARS method, though step size is small. In particular, in up-and-out barrier option, the prices of LARSI method converge uniformly, while those in LARS method oscillate. Therefore, we can think that LARSI method is efficient in valuing the up-and-out barrier option and compensate the defect of LARS method.

Tables 12-15 show the price of the double barrier option with different numbers of time steps. The lower barrier is set as 70 and the upper barrier is set as 150 . The prices of the double barrier option decrease progressively and oscillate. In double barrier option, the convergence patterns 
TABLE 6: Pricing the American down-and-out barrier call option with the LARS-type method.

\begin{tabular}{|c|c|c|c|c|c|c|c|c|c|c|c|c|}
\hline \multirow{3}{*}{$N$} & \multicolumn{6}{|c|}{ LARS method } & \multicolumn{6}{|c|}{ LARSI method } \\
\hline & \multicolumn{3}{|c|}{ Regime 1} & \multicolumn{3}{|c|}{ Regime 2} & \multicolumn{3}{|c|}{ Regime 1} & \multicolumn{3}{|c|}{ Regime 2} \\
\hline & Price & Difference & Ratio & Price & Difference & Ratio & Price & Difference & Ratio & Price & Difference & Ratio \\
\hline 20 & 11.6335 & -1.4914 & -0.1840 & 13.4120 & -2.1980 & -0.1660 & 8.7988 & -0.0745 & -2.1090 & 9.5653 & -0.0680 & -1.8901 \\
\hline 40 & 10.1420 & 0.2745 & -1.0490 & 11.2140 & 0.3648 & -1.0776 & 8.7243 & 0.1571 & 0.3370 & 9.4974 & 0.1285 & 0.3463 \\
\hline 80 & 10.4165 & -0.2879 & 2.1678 & 11.5788 & -0.3931 & 2.0738 & 8.8814 & 0.0529 & 0.0803 & 9.6258 & 0.0445 & 0.0582 \\
\hline 160 & 10.1286 & -0.6242 & 0.0853 & 11.1857 & -0.8152 & 0.0839 & 8.9343 & 0.0042 & 3.8905 & 9.6703 & 0.0026 & 5.3210 \\
\hline 320 & 9.5043 & -0.0533 & -0.9695 & 10.3705 & -0.0684 & -0.9553 & 8.9386 & 0.0165 & 0.8524 & 9.6729 & 0.0138 & 0.8811 \\
\hline 640 & 9.4511 & 0.0516 & -8.5059 & 10.3021 & 0.0654 & -8.4494 & 8.9551 & 0.0141 & -0.1603 & 9.6867 & 0.0121 & -0.1824 \\
\hline 1280 & 9.5027 & -0.4392 & -0.3926 & 10.3674 & -0.5523 & -0.3892 & 8.9692 & -0.0023 & -1.0424 & 9.6988 & -0.0022 & -0.8923 \\
\hline 2560 & 9.0635 & 0.1724 & & 9.8151 & 0.2150 & & 8.9669 & 0.0024 & & 9.6966 & 0.0020 & \\
\hline 5120 & 9.2359 & & & 10.0301 & & & 8.9693 & & & 9.6986 & & \\
\hline
\end{tabular}

TABLE 7: Pricing the American down-and-out barrier put option with the LARS-type method.

\begin{tabular}{|c|c|c|c|c|c|c|c|c|c|c|c|c|}
\hline \multirow{3}{*}{$N$} & \multicolumn{6}{|c|}{ LARS method } & \multicolumn{6}{|c|}{ LARSI method } \\
\hline & \multicolumn{3}{|c|}{ Regime 1} & \multicolumn{3}{|c|}{ Regime 2} & \multicolumn{3}{|c|}{ Regime 1} & \multicolumn{3}{|c|}{ Regime 2} \\
\hline & Price & Difference & Ratio & Price & Difference & Ratio & Price & Difference & Ratio & Price & Difference & Ratio \\
\hline 20 & 6.3763 & -1.3074 & -1.0040 & 6.8947 & -1.5517 & -0.9916 & 0.8840 & 2.1826 & 0.6745 & 0.9394 & 2.2911 & 0.6740 \\
\hline 40 & 5.0690 & 1.3126 & 0.1710 & 5.3429 & 1.5387 & 0.1778 & 3.0667 & 1.4722 & 0.5649 & 3.2305 & 1.5442 & 0.5954 \\
\hline 80 & 6.3816 & 0.2245 & -0.9941 & 6.8817 & 0.2736 & -1.0116 & 4.5389 & 0.8316 & 0.5833 & 4.7747 & 0.9195 & 0.6112 \\
\hline 160 & 6.6061 & -0.2232 & -1.0030 & 7.1553 & -0.2768 & -0.9936 & 5.3705 & 0.4851 & 0.6695 & 5.6942 & 0.5620 & 0.6775 \\
\hline 320 & 6.3829 & 0.2239 & 0.9866 & 6.8785 & 0.2750 & 1.0062 & 5.8556 & 0.3247 & 0.6666 & 6.2561 & 0.3807 & 0.6768 \\
\hline 640 & 6.6067 & 0.2209 & -0.9993 & 7.1535 & 0.2767 & -1.0017 & 6.1803 & 0.2165 & 0.6214 & 6.6369 & 0.2577 & 0.6431 \\
\hline 1280 & 6.8276 & -0.2207 & -1.0003 & 7.4302 & -0.2772 & -0.9991 & 6.3968 & 0.1345 & 0.7080 & 6.8945 & 0.1657 & 0.7073 \\
\hline 2560 & 6.6069 & 0.2208 & & 7.1530 & 0.2769 & & 6.5313 & 0.0952 & & 7.0603 & 0.1172 & \\
\hline 5120 & 6.8277 & & & 7.4299 & & & 6.6265 & & & 7.1775 & & \\
\hline
\end{tabular}

TABLE 8: Pricing the European up-and-out barrier call option with the LARS-type method.

\begin{tabular}{|c|c|c|c|c|c|c|c|c|c|c|c|c|}
\hline \multirow{3}{*}{$N$} & \multicolumn{6}{|c|}{ LARS method } & \multicolumn{6}{|c|}{ LARSI method } \\
\hline & \multicolumn{3}{|c|}{ Regime 1} & \multicolumn{3}{|c|}{ Regime 2} & \multicolumn{3}{|c|}{ Regime 1} & \multicolumn{3}{|c|}{ Regime 2} \\
\hline & Price & Difference & Ratio & Price & Difference & Ratio & Price & Difference & Ratio & Price & Difference & Ratio \\
\hline 20 & 0.6005 & -0.4275 & -0.1184 & 0.3355 & -0.2417 & -0.1210 & 0.0478 & 0.0329 & -0.8215 & 0.0255 & 0.0182 & -0.8136 \\
\hline 40 & 0.1730 & 0.0506 & -3.2236 & 0.0938 & 0.0293 & -3.0933 & 0.0808 & -0.0271 & -0.0449 & 0.0437 & -0.0148 & -0.0481 \\
\hline 80 & 0.2236 & -0.1631 & -0.3359 & 0.1230 & -0.0905 & -0.3348 & 0.0537 & 0.0012 & -0.5066 & 0.0289 & 0.0007 & -0.3764 \\
\hline 160 & 0.0604 & 0.0548 & -0.9761 & 0.0326 & 0.0303 & -0.9728 & 0.0549 & -0.0006 & -0.5856 & 0.0296 & -0.0003 & -0.8087 \\
\hline 320 & 0.1152 & -0.0535 & -0.2984 & 0.0629 & -0.0295 & -0.2982 & 0.0543 & 0.0004 & -1.5676 & 0.0293 & 0.0002 & -1.3799 \\
\hline 640 & 0.0617 & 0.0160 & -0.9796 & 0.0334 & 0.0088 & -0.9766 & 0.0547 & -0.0006 & -0.0076 & 0.0295 & -0.0003 & -0.0253 \\
\hline 1280 & 0.0777 & -0.0156 & -0.0301 & 0.0422 & -0.0086 & -0.0306 & 0.0541 & 0.0000 & -52.8157 & 0.0292 & 0.0000 & -16.3714 \\
\hline 2560 & 0.0621 & 0.0004 & & 0.0336 & 0.0002 & & 0.0541 & -0.0002 & & 0.0292 & -0.0001 & \\
\hline 5120 & 0.0625 & & & 0.0338 & & & 0.0539 & & & 0.0291 & & \\
\hline
\end{tabular}

between LARS and LARSI methods are similar but there is small difference. In European double barrier option, the prices obtained by LARSI method also demonstrate better stability compared with LARS method. On the other hand, in American double barrier option, LARS method gives a more stable performance than LARSI method. Therefore, we can use both LARS and LARSI methods to price double barrier options efficiently.

We now consider a few more examples. First, we compare the prices between different barrier levels in double barrier option. Table 16 summarizes the value of the European double barrier options with different barrier levels (lower barriers of $90,80,70,60$, and 50 and upper barriers of 110, 120, 130, 140, and 150) using 1000 time steps. When the difference between the upper barrier and lower barrier is smaller, the prices of the options are lower as there is a bigger chance of crossing the barrier and becoming out of value. The effect of barriers is more significant for regime 2 because the underlying asset with higher volatility in regime 2 have a greater chance of reaching the barrier level. When the difference between the 
TABLE 9: Pricing the European up-and-out barrier put option with the LARS-type method.

\begin{tabular}{|c|c|c|c|c|c|c|c|c|c|c|c|c|}
\hline \multirow{3}{*}{$N$} & \multicolumn{6}{|c|}{ LARS method } & \multicolumn{6}{|c|}{ LARSI method } \\
\hline & \multicolumn{3}{|c|}{ Regime 1} & \multicolumn{3}{|c|}{ Regime 2} & \multicolumn{3}{|c|}{ Regime 1} & \multicolumn{3}{|c|}{ Regime 2} \\
\hline & Price & Difference & Ratio & Price & Difference & Ratio & Price & Difference & Ratio & Price & Difference & Ratio \\
\hline 20 & 7.5614 & -1.0591 & -0.1951 & 8.5033 & -1.4915 & -0.1726 & 5.2933 & -0.1666 & -1.1509 & 5.5685 & -0.1550 & -1.0705 \\
\hline 40 & 6.5023 & 0.2066 & -5.9780 & 7.0118 & 0.2575 & -5.8889 & 5.1267 & 0.1918 & -0.0217 & 5.4135 & 0.1659 & -0.0306 \\
\hline 80 & 6.7089 & -1.2352 & -0.4839 & 7.2693 & -1.5164 & -0.4677 & 5.3184 & -0.0042 & -3.5735 & 5.5794 & -0.0051 & -2.3325 \\
\hline 160 & 5.4737 & 0.5977 & -0.9975 & 5.7530 & 0.7092 & -1.0004 & 5.3143 & 0.0149 & -0.0632 & 5.5744 & 0.0118 & -0.1022 \\
\hline 320 & 6.0715 & -0.5963 & -0.3598 & 6.4622 & -0.7095 & -0.3538 & 5.3291 & -0.0009 & -6.2294 & 5.5862 & -0.0012 & -4.0389 \\
\hline 640 & 5.4752 & 0.2145 & -0.9983 & 5.7527 & 0.2510 & -1.0003 & 5.3282 & 0.0059 & 0.0862 & 5.5850 & 0.0049 & 0.0695 \\
\hline 1280 & 5.6897 & -0.2142 & -0.0297 & 6.0037 & -0.2511 & -0.0291 & 5.3340 & 0.0005 & 3.6497 & 5.5899 & 0.0003 & 4.6204 \\
\hline 2560 & 5.4756 & 0.0064 & & 5.7526 & 0.0073 & & 5.3346 & 0.0018 & & 5.5902 & 0.0016 & \\
\hline 5120 & 5.4819 & & & 5.7599 & & & 5.3364 & & & 5.5918 & & \\
\hline
\end{tabular}

TABLE 10: Pricing the American up-and-out barrier call option with the LARS-type method.

\begin{tabular}{|c|c|c|c|c|c|c|c|c|c|c|c|c|}
\hline \multirow{3}{*}{$N$} & \multicolumn{6}{|c|}{ LARS method } & \multicolumn{6}{|c|}{ LARSI method } \\
\hline & \multicolumn{3}{|c|}{ Regime 1} & \multicolumn{3}{|c|}{ Regime 2} & \multicolumn{3}{|c|}{ Regime 1} & \multicolumn{3}{|c|}{ Regime 2} \\
\hline & Price & Difference & Ratio & Price & Difference & Ratio & Price & Difference & Ratio & Price & Difference & Ratio \\
\hline 20 & 7.1590 & -1.6580 & -0.9986 & 7.5712 & -1.8630 & -0.9921 & 0.1801 & 2.2234 & 0.8266 & 0.1714 & 2.3199 & 0.8042 \\
\hline 40 & 5.5009 & 1.6556 & -1.0003 & 5.7082 & 1.8482 & -1.0019 & 2.4035 & 1.8378 & 0.5322 & 2.4913 & 1.8656 & 0.5625 \\
\hline 80 & 7.1565 & -1.6561 & -0.9997 & 7.5564 & -1.8517 & -0.9981 & 4.2413 & 0.9781 & 0.6761 & 4.3569 & 1.0495 & 0.6833 \\
\hline 160 & 5.5504 & 1.6556 & -0.3718 & 5.7047 & 1.8482 & -0.3781 & 5.2195 & 0.6613 & 0.6434 & 5.4064 & 0.7171 & 0.6578 \\
\hline 320 & 7.1560 & -0.6155 & -0.9998 & 7.5528 & -0.6988 & -0.9987 & 5.8808 & 0.4255 & 0.6971 & 6.1235 & 0.4717 & 0.6999 \\
\hline 640 & 6.5405 & 0.6154 & -0.2311 & 6.8541 & 0.6979 & -0.2333 & 6.3063 & 0.2966 & 0.6693 & 6.5952 & 0.3301 & 0.6781 \\
\hline 1280 & 7.1559 & -0.1422 & -0.9998 & 7.5520 & -0.1628 & -0.9986 & 6.6030 & 0.1985 & 0.7010 & 6.9253 & 0.2238 & 0.7028 \\
\hline 2560 & 7.0136 & 0.1423 & & 7.3892 & 0.1625 & & 6.8015 & 0.1391 & & 7.1491 & 0.1573 & \\
\hline 5120 & 7.1559 & & & 7.5517 & & & 6.9406 & & & 7.3064 & & \\
\hline
\end{tabular}

TABLE 11: Pricing the American up-and-out barrier put option with the LARS-type method.

\begin{tabular}{|c|c|c|c|c|c|c|c|c|c|c|c|c|}
\hline \multirow{3}{*}{$N$} & \multicolumn{6}{|c|}{ LARS method } & \multicolumn{6}{|c|}{ LARSI method } \\
\hline & \multicolumn{3}{|c|}{ Regime 1} & \multicolumn{3}{|c|}{ Regime 2} & \multicolumn{3}{|c|}{ Regime 1} & \multicolumn{3}{|c|}{ Regime 2} \\
\hline & Price & Difference & Ratio & Price & Difference & Ratio & Price & Difference & Ratio & Price & Difference & Ratio \\
\hline 20 & 7.9544 & -1.0884 & -0.1976 & 9.0574 & -1.5517 & -0.1724 & 5.6154 & -0.1700 & -1.1779 & 5.9979 & -0.1575 & -1.1179 \\
\hline 40 & 6.8660 & 0.2151 & -5.9265 & 7.5057 & 0.2674 & -5.8956 & 5.4454 & 0.2002 & -0.0200 & 5.8404 & 0.1760 & -0.0338 \\
\hline 80 & 7.0811 & -1.2748 & -0.4846 & 7.7732 & -1.5768 & -0.4676 & 5.6456 & -0.0040 & -3.9377 & 6.0165 & -0.0059 & -2.0446 \\
\hline 160 & 5.8064 & 0.6177 & -0.9968 & 6.1964 & 0.7373 & -1.0009 & 5.6416 & 0.0158 & -0.0564 & 6.0105 & 0.0121 & -0.1183 \\
\hline 320 & 6.4241 & -0.6157 & -0.3600 & 6.9337 & -0.7380 & -0.3537 & 5.6574 & -0.0009 & -6.8705 & 6.0227 & -0.0014 & -3.5386 \\
\hline 640 & 5.8084 & 0.2216 & -0.9980 & 6.1957 & 0.2611 & -1.0004 & 5.6565 & 0.0061 & 0.0889 & 6.0212 & 0.0051 & 0.0687 \\
\hline 1280 & 6.0300 & -0.2212 & -0.0297 & 6.4568 & -0.2612 & -0.0291 & 5.6626 & 0.0005 & 3.5307 & 6.0263 & 0.0003 & 4.6524 \\
\hline 2560 & 5.8088 & 0.0066 & & 6.1956 & 0.0076 & & 5.6631 & 0.0019 & & 6.0267 & 0.0016 & \\
\hline 5120 & 5.8154 & & & 6.2032 & & & 5.6650 & & & 6.0283 & & \\
\hline
\end{tabular}

barriers increases, its effect on the barrier options is reduced. Also, the options with a larger volatility in regime 2 have a higher price than the same options in regime 1 . Their prices are lower than those of the European call option, which has prices of 12.7587 and 15.7677 with respect to the two regimes, respectively. And the prices of LARSI method in the two regimes are closer to LARS method.

Second, we predict that the convergence rate of the proposed model will be harmed if the volatility of different regimes is largely different from each regime to another. All the other conditions are assumed to be the same, but the volatility of the two regimes becomes 0.10 and 0.50 . The prices of the European call option are tested (Table 17). The transition probabilities of regime 1 with 20 time steps in the three branches are $0.0224,0.9689$, and 0.0086 , respectively. Note that most of the probabilities are distributed on the middle branch. On the other hand, the transition probabilities of regime 2 with 20 time steps in the three branches are 0.3754 , 
TABLE 12: Pricing the European double barrier call option with the LARS-type method.

\begin{tabular}{|c|c|c|c|c|c|c|c|c|c|c|c|c|}
\hline \multirow{3}{*}{$N$} & \multicolumn{6}{|c|}{ LARS method } & \multicolumn{6}{|c|}{ LARSI method } \\
\hline & & Regime 1 & & & Regime 2 & & & Regime 1 & & & Regime 2 & \\
\hline & Price & Difference & Ratio & Price & Difference & Ratio & Price & Difference & Ratio & Price & Difference & Ratio \\
\hline 20 & 7.4788 & -0.0969 & 12.0395 & 5.9400 & -0.0507 & 24.3064 & 5.6723 & 0.0724 & 0.3143 & 4.1254 & 0.0460 & 0.8110 \\
\hline 40 & 7.3819 & -1.1669 & -0.2170 & 5.8893 & -1.2334 & -0.1959 & 5.7447 & 0.0228 & 0.6148 & 4.1714 & 0.0373 & 0.3820 \\
\hline 80 & 6.2150 & 0.2532 & -0.9646 & 4.6559 & 0.2416 & -0.9230 & 5.7675 & 0.0140 & 0.4757 & 4.2086 & 0.0142 & 0.4819 \\
\hline 160 & 6.4682 & -0.2442 & 0.9075 & 4.8975 & -0.2230 & 1.0357 & 7.7815 & 0.0067 & 0.5439 & 4.2229 & 0.0069 & 0.4879 \\
\hline 320 & 6.2240 & -0.2216 & 0.5402 & 4.6745 & -0.2310 & 0.5033 & 5.7881 & 0.0036 & 0.5865 & 4.2297 & 0.0033 & 0.4995 \\
\hline 640 & 6.0024 & -0.1197 & -1.0097 & 4.4435 & -0.1163 & -1.0204 & 5.7918 & 0.0021 & 0.4641 & 4.2331 & 0.0017 & 0.5644 \\
\hline 1280 & 5.8827 & 0.1209 & -0.9951 & 4.3273 & 0.1186 & -0.9899 & 5.7939 & 0.0010 & 0.2764 & 4.2348 & 0.0009 & 0.5660 \\
\hline 2560 & 6.0035 & -0.1202 & & 4.4459 & -0.1174 & & 5.7949 & 0.0002 & & 4.2357 & 0.0005 & \\
\hline 5120 & 5.8833 & & & 4.3285 & & & 5.7951 & & & 4.2362 & & \\
\hline
\end{tabular}

TABLE 13: Pricing the European double barrier put option with the LARS-type method.

\begin{tabular}{|c|c|c|c|c|c|c|c|c|c|c|c|c|}
\hline \multirow{3}{*}{$N$} & \multicolumn{6}{|c|}{ LARS method } & \multicolumn{6}{|c|}{ LARSI method } \\
\hline & & Regime 1 & & & Regime 2 & & & Regime 1 & & & Regime 2 & \\
\hline & Price & Difference & Ratio & Price & Difference & Ratio & Price & Difference & Ratio & Price & Difference & Ratio \\
\hline 20 & 12.6678 & 0.9570 & -0.9571 & 9.2339 & 1.0463 & -0.9747 & 11.8281 & 0.0825 & 0.5818 & 8.2848 & 0.1559 & 0.3274 \\
\hline 40 & 13.6248 & -0.9160 & 0.4357 & 10.2802 & -1.0198 & 0.3518 & 11.9106 & 0.0480 & 0.6612 & 8.4407 & 0.0511 & 0.6639 \\
\hline 80 & 12.7088 & -0.3991 & -1.0572 & 9.2604 & -0.3587 & -1.1282 & 11.9586 & 0.0317 & 0.3365 & 8.4918 & 0.0339 & 0.4967 \\
\hline 160 & 12.3098 & 0.4219 & -1.0389 & 8.9017 & 0.4047 & -1.1260 & 11.9903 & 0.0107 & 0.6280 & 8.5257 & 0.0168 & 0.5402 \\
\hline 320 & 12.7317 & -0.4383 & 0.1409 & 9.3064 & -0.4557 & 0.1572 & 12.0010 & 0.0067 & 0.5624 & 8.5425 & 0.0091 & 0.5195 \\
\hline 640 & 12.2934 & -0.0617 & -1.0474 & 8.8507 & -0.0716 & -1.0811 & 12.0077 & 0.00368 & 0.5285 & 8.5516 & 0.0047 & 0.4499 \\
\hline 1280 & 12.2316 & 0.0647 & -0.9775 & 8.7791 & 0.0774 & -0.9625 & 12.0115 & 0.0020 & 0.3737 & 8.5563 & 0.0021 & 0.3817 \\
\hline 2560 & 12.2963 & -0.0632 & & 8.8565 & -0.0745 & & 12.0135 & 0.0007 & & 8.5585 & 0.0008 & \\
\hline 5120 & 12.2331 & & & 8.7820 & & & 12.0142 & & & 8.5593 & & \\
\hline
\end{tabular}

TABle 14: Pricing the American double barrier call option with the LARS-type method.

\begin{tabular}{|c|c|c|c|c|c|c|c|c|c|c|c|c|}
\hline \multirow{3}{*}{$N$} & \multicolumn{6}{|c|}{ LARS method } & \multicolumn{6}{|c|}{ LARSI method } \\
\hline & & Regime 1 & & & Regime 2 & & & Regime 1 & & & Regime 2 & \\
\hline & Price & Difference & Ratio & Price & Difference & Ratio & Price & Difference & Ratio & Price & Difference & Ratio \\
\hline 20 & 12.4324 & 0.0648 & -1.2446 & 14.9452 & 0.1245 & -1.7594 & 12.0743 & 0.1873 & 0.4084 & 14.2092 & 0.3145 & 0.4477 \\
\hline 40 & 12.4972 & -0.0807 & -0.6282 & 15.0697 & -0.2190 & -0.3987 & 12.2616 & 0.0765 & 0.6891 & 14.5237 & 0.1408 & 0.7128 \\
\hline 80 & 12.4165 & 0.0507 & 0.1797 & 14.8506 & 0.0873 & 0.3136 & 12.3381 & 0.0527 & 0.5628 & 14.6645 & 0.1003 & 0.5815 \\
\hline 160 & 12.4672 & 0.0091 & -1.2180 & 14.9380 & 0.0274 & -1.4265 & 12.3908 & 0.0297 & 0.6462 & 14.7648 & 0.0584 & 0.6694 \\
\hline 320 & 12.4763 & -0.0111 & -0.0077 & 14.9654 & -0.0391 & 0.0678 & 12.4204 & 0.0192 & 0.6734 & 14.8232 & 0.0391 & 0.6892 \\
\hline 640 & 12.4653 & 0.0001 & 218.2816 & 14.9263 & -0.0026 & -15.9678 & 12.4396 & 0.0129 & 0.6694 & 14.8622 & 0.0269 & 0.6824 \\
\hline 1280 & 12.4653 & 0.0187 & -0.2806 & 14.9236 & 0.0423 & -0.3198 & 12.4525 & 0.0086 & 0.6627 & 14.8891 & 0.0184 & 0.6721 \\
\hline 2560 & 12.4840 & -0.0052 & & 14.9659 & -0.0135 & & 12.4611 & 0.0058 & & 14.9075 & 0.0124 & \\
\hline 5120 & 12.4788 & & & 14.9524 & & & 12.4669 & & & 14.9199 & & \\
\hline
\end{tabular}

TABle 15: Pricing the American double barrier put option with the LARS-type method.

\begin{tabular}{|c|c|c|c|c|c|c|c|c|c|c|c|c|}
\hline \multirow{3}{*}{$N$} & \multicolumn{6}{|c|}{ LARS method } & \multicolumn{6}{|c|}{ LARSI method } \\
\hline & & Regime 1 & & & Regime 2 & & & Regime 1 & & & Regime 2 & \\
\hline & Price & Difference & Ratio & Price & Difference & Ratio & Price & Difference & Ratio & Price & Difference & Ratio \\
\hline 20 & 21.8204 & 0.0549 & -0.6749 & 23.4097 & -0.0197 & 8.4024 & 21.7067 & 0.0826 & 0.1183 & 23.0915 & 0.0456 & -0.2495 \\
\hline 40 & 21.8754 & -0.0371 & -0.5947 & 23.3900 & -0.1657 & -0.2468 & 21.7893 & 0.0098 & 1.4698 & 23.1372 & -0.0114 & -0.9766 \\
\hline 80 & 21.8383 & 0.0221 & -0.4942 & 23.2243 & 0.0409 & -1.0437 & 21.7991 & 0.0144 & 0.3350 & 23.1258 & 0.0111 & 0.1763 \\
\hline 160 & 21.8603 & -0.0109 & 1.1220 & 23.2652 & -0.0427 & 0.9272 & 21.8135 & 0.0048 & 0.5809 & 23.1369 & 0.0020 & 0.7872 \\
\hline 320 & 21.8494 & -0.0122 & 0.6107 & 23.2225 & -0.0396 & 0.5892 & 21.8183 & 0.0028 & 0.6598 & 23.1389 & 0.0015 & 0.8910 \\
\hline 640 & 21.8372 & -0.0075 & -1.1900 & 23.1829 & -0.0233 & -0.9767 & 21.8211 & 0.0018 & 0.4232 & 23.1404 & 0.0014 & 0.3589 \\
\hline 1280 & 21.8297 & 0.0089 & -0.9289 & 23.1596 & 0.0228 & -1.0140 & 21.8229 & 0.0008 & 0.2702 & 23.1418 & 0.0005 & -0.2092 \\
\hline 2560 & 21.8386 & -0.0082 & & 23.1824 & -0.0231 & & 21.8237 & 0.0002 & & 23.1423 & -0.0001 & \\
\hline 5120 & 21.8304 & & & 23.1593 & & & 21.8239 & & & 23.1422 & & \\
\hline
\end{tabular}


TABLE 16: Price of the European double barrier call options with different barrier levels: LARS-type method.

\begin{tabular}{lcccccccccc}
\hline & \multicolumn{9}{c}{ LARS method } & \multicolumn{3}{c}{ LARSI method } \\
& 90 & 80 & 70 & 60 & 50 & 90 & 80 & 70 & 60 & 50 \\
\hline Regime 1 & & & & & & & & & \\
110 & 0.0016 & 0.0397 & 0.0746 & 0.0791 & 0.0793 & $7.1335 e-04$ & 0.0248 & 0.0498 & 0.0542 & 0.0544 \\
120 & 0.1113 & 0.4554 & 0.6065 & 0.6210 & 0.6218 & 0.1028 & 0.4304 & 0.5759 & 0.5941 & 0.5949 \\
130 & 0.7303 & 1.6610 & 1.9502 & 1.9741 & 1.9753 & 0.7090 & 1.6224 & 1.9079 & 1.9387 & 1.9400 \\
140 & 2.0142 & 3.5940 & 3.9971 & 4.0276 & 4.0290 & 1.8812 & 3.4030 & 3.7974 & 3.8365 & 3.8381 \\
150 & 3.3872 & 5.4423 & 5.9130 & 5.9469 & 5.9485 & 3.2997 & 5.3257 & 5.7937 & 5.8378 & 5.8395 \\
Regime 2 & & & & & & & & & \\
110 & $1.2048 e-04$ & 0.0086 & 0.0322 & 0.0416 & 0.0430 & $4.9945 e-05$ & 0.0050 & 0.0201 & 0.0282 & 0.0294 \\
120 & 0.0174 & 0.1552 & 0.3127 & 0.3537 & 0.3584 & 0.0159 & 0.1446 & 0.2899 & 0.3374 & 0.3422 \\
130 & 0.2009 & 0.7600 & 1.1536 & 1.2335 & 1.2412 & 0.1933 & 0.7364 & 1.1129 & 1.2087 & 1.2169 \\
140 & 0.8040 & 2.0252 & 2.6775 & 2.7907 & 2.8005 & 0.7321 & 1.8843 & 2.4997 & 2.6349 & 2.6454 \\
150 & 1.6795 & 3.5227 & 4.3689 & 4.5025 & 4.5133 & 1.6185 & 3.4164 & 4.2349 & 4.3980 & 4.4097 \\
\hline
\end{tabular}

TABLE 17: Pricing the European call option with the LARS method: great deviation in volatility.

\begin{tabular}{|c|c|c|c|c|c|c|}
\hline \multirow{2}{*}{$N$} & \multicolumn{3}{|c|}{ Regime 1} & \multicolumn{3}{|c|}{ Regime 2} \\
\hline & Price & Difference & Ratio & Price & Difference & Ratio \\
\hline 20 & 9.6172 & 0.0900 & 0.6695 & 20.2019 & -0.1418 & 0.4953 \\
\hline 40 & 9.7071 & 0.0602 & 0.4450 & 20.0601 & -0.0702 & 0.4968 \\
\hline 80 & 9.7674 & 0.0268 & 0.4514 & 19.9899 & -0.0349 & 0.4984 \\
\hline 160 & 9.7942 & 0.0121 & 0.4825 & 19.9550 & -0.0174 & 0.4993 \\
\hline 320 & 9.8063 & 0.0058 & 0.4925 & 19.9376 & -0.0087 & 0.4996 \\
\hline 640 & 9.8121 & 0.0029 & 0.4965 & 19.9289 & -0.0043 & 0.4998 \\
\hline 1280 & 9.8150 & 0.0014 & 0.4983 & 19.9246 & -0.0022 & 0.4999 \\
\hline 2560 & 9.8164 & 0.0007 & & 19.9224 & -0.0011 & \\
\hline 5120 & 9.8171 & & & 19.9214 & & \\
\hline
\end{tabular}

0.2235 , and 0.4010 , respectively. The value of the European option is positively related to the volatility and so the price in regime 1 decreases, while the price in regime 2 increases, when we compare the results with the previous numerical experiments. The pricing error in regime 1 is larger when we compare it with the numerical results in the previous example, since large $\sigma$ is used in this lattice.

Figures 1-6 show the comparison of the convergence behaviors of various trinomial tree methods in pricing of options with barrier. In this experiment, "Basic" denotes the method of Y\&Y, "LA" is the local average trinomial tree method, "LA interpolation" is the combination of local average trinomial tree method and interpolation method, "LA-RS" means the LARS method, and "LA-RS interpolation" means the LARSI method. LARSI method gives much closer values at smaller number of steps than other models in pricing various barrier options. The results show that the prices of options with barrier by LARSI method converge smoothly always faster than those of the other trinomial tree methods. As a result, LARSI method is more efficient than other models in pricing barrier options. The main reason is that LARSI method can fit derivatives' specifications to suppress the oscillation problem.

\section{Concluding Remark}

Regime switching model is one of popular models in finance area. We develop the trinomial tree method based on regime switching method and local averages of the option price and compare its performance with other trinomial tree schemes in terms of their accuracy and efficiency. We also modify the LARS method using interpolation method and show that it works very well for general types of options with barrier including European type and American type. The LARSI method has a smoothing effect to reduce oscillations of the tree method, and it seems to accelerate the convergence rate. Finally, we can find that LARSI method provides good performance for valuing options with barrier in a regime switching model.

\section{Appendix}

\section{Approximation for Exact Pricing Formula of the Options with Regime Switching}

We assume that $f$ is a function continuous and $n$ times differentiable in an interval $\Omega=[a, b]$ and $a<x-3 h<$ $x-2 h<\cdots<x+2 h<x+3 h<b$ for some $x \in \Omega$ and $h>0$. 


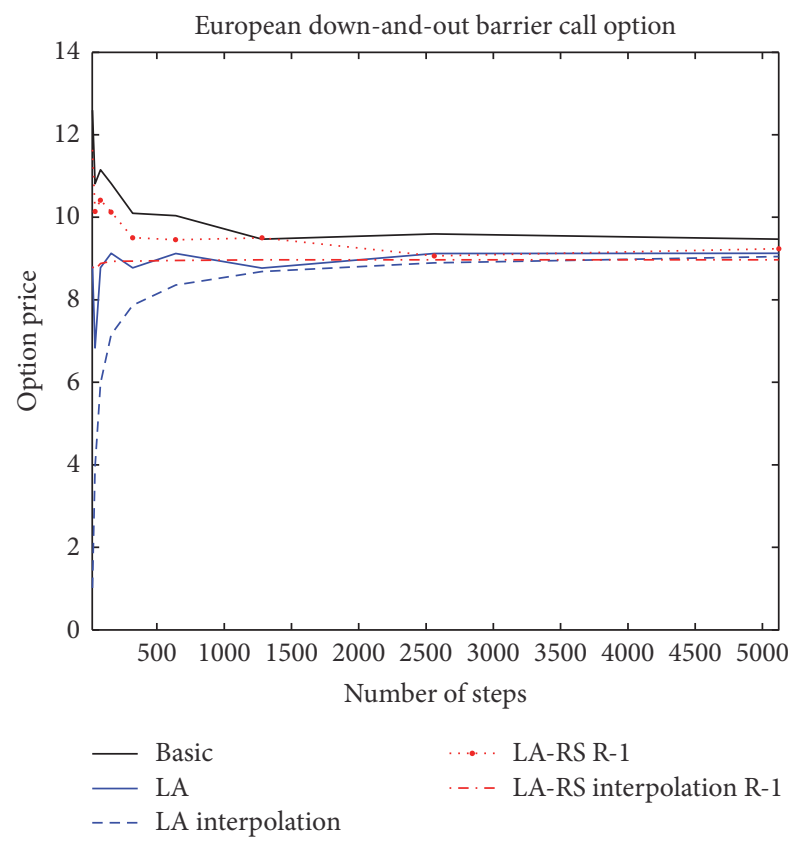

(a) European down-and-out barrier call option, regime 1

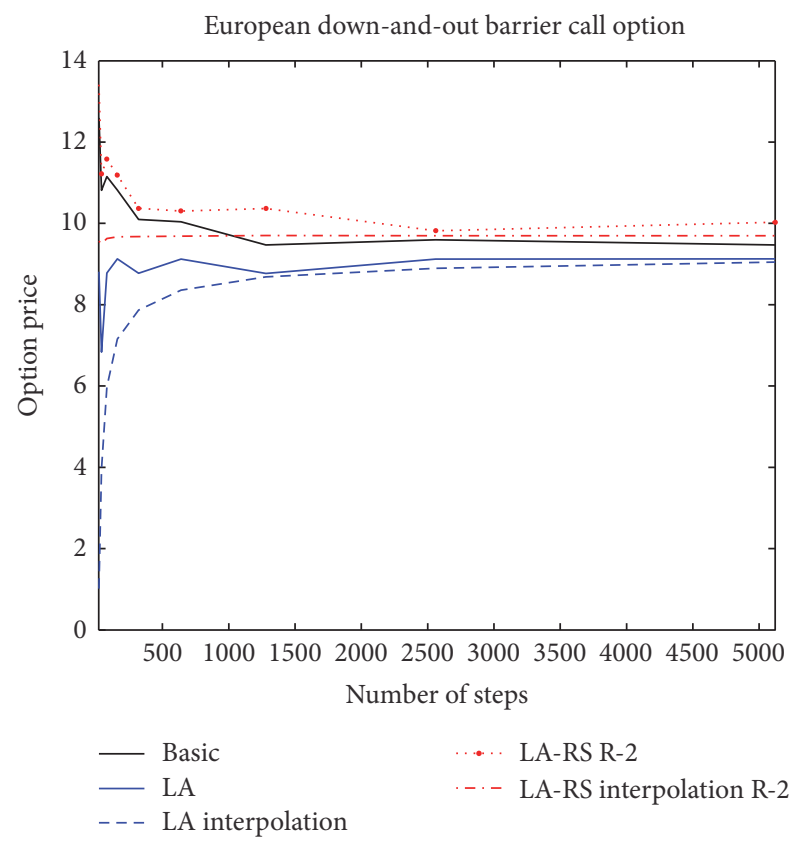

(b) European down-and-out barrier call option, regime 2

FIgURE 1: Convergence of various models for calculation of European down-and-out barrier call option.

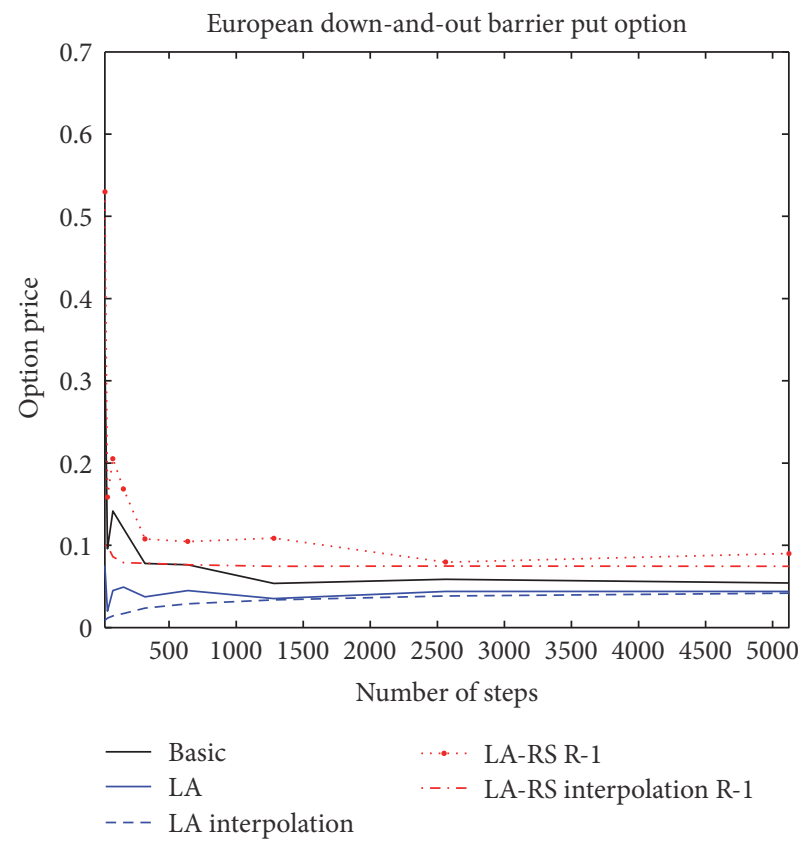

(a) European down-and-out barrier put option, regime 1

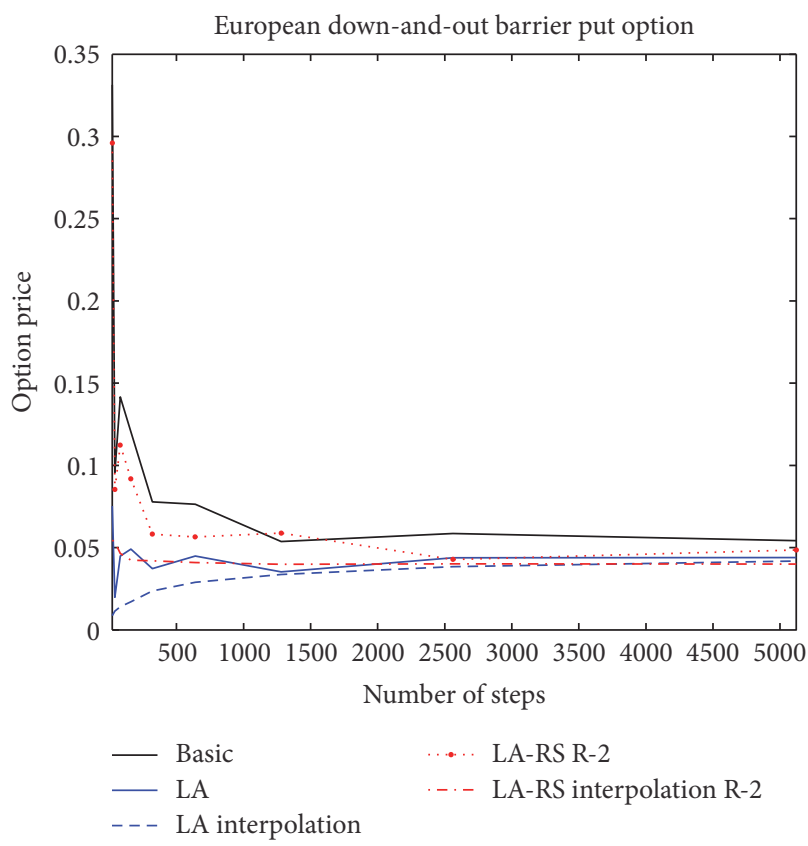

(b) European down-and-out barrier put option, regime 2

FIGURE 2: Convergence of various models for calculation of European down-and-out barrier put option.

Let us denote $f_{j}=f(x+j h)$ for $j \in \mathbb{R}$ and consider the following equation:

$$
\begin{aligned}
f_{0}^{\prime} & +y_{1}\left(f_{1}^{\prime}+f_{-1}^{\prime}\right)+y_{2}\left(f_{2}^{\prime}+f_{-2}^{\prime}\right) \\
& =z_{1} \frac{f_{1}-f_{-1}}{2 h}+z_{2} \frac{f_{2}-f_{-2}}{4 h}+z_{3} \frac{f_{3}-f_{-3}}{6 h}
\end{aligned}
$$

where $y_{1}, y_{2}, z_{1}, z_{2}$, and $z_{3}$ are constants. Then, from the Taylor series, (A.1) can be represented as

$$
\begin{aligned}
f_{0}^{\prime} & +y_{1}\left(f_{1}^{\prime}+f_{-1}^{\prime}\right)+y_{2}\left(f_{2}^{\prime}+f_{-2}^{\prime}\right) \\
& =\left(1+2 y_{1}+2 y_{2}\right) f_{0}^{\prime}+\left(2 y_{1}+8 y_{2}\right) \frac{h^{2}}{2} f_{0}^{(3)}+\cdots,
\end{aligned}
$$




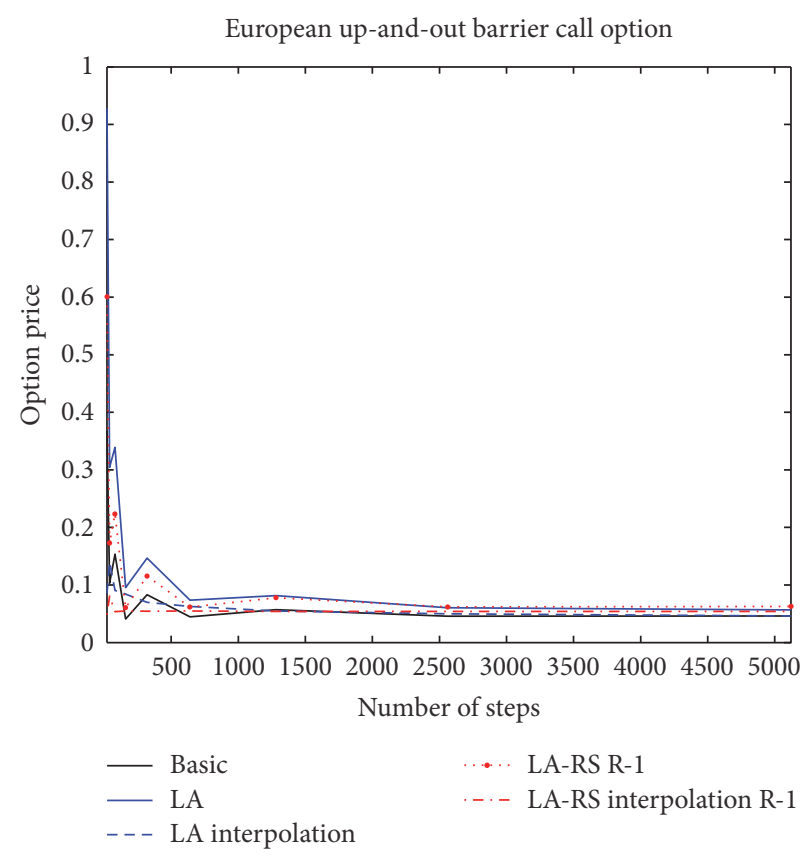

(a) European up-and-out barrier call option, regime 1

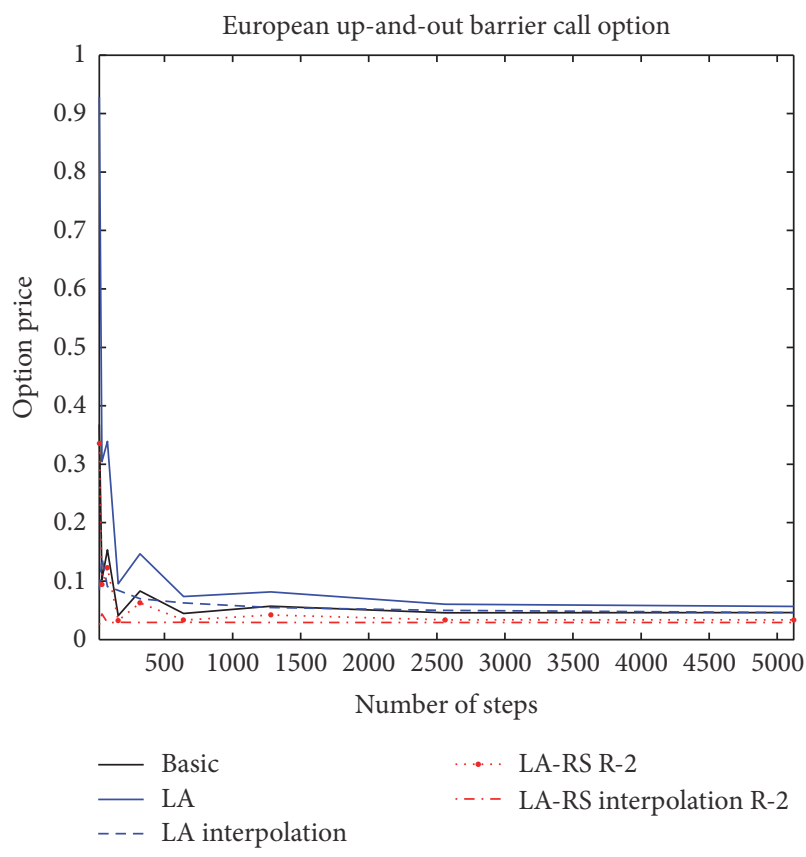

(b) European up-and-out barrier call option, regime 2

FIGURE 3: Convergence of various models for calculation of European up-and-out barrier call option.

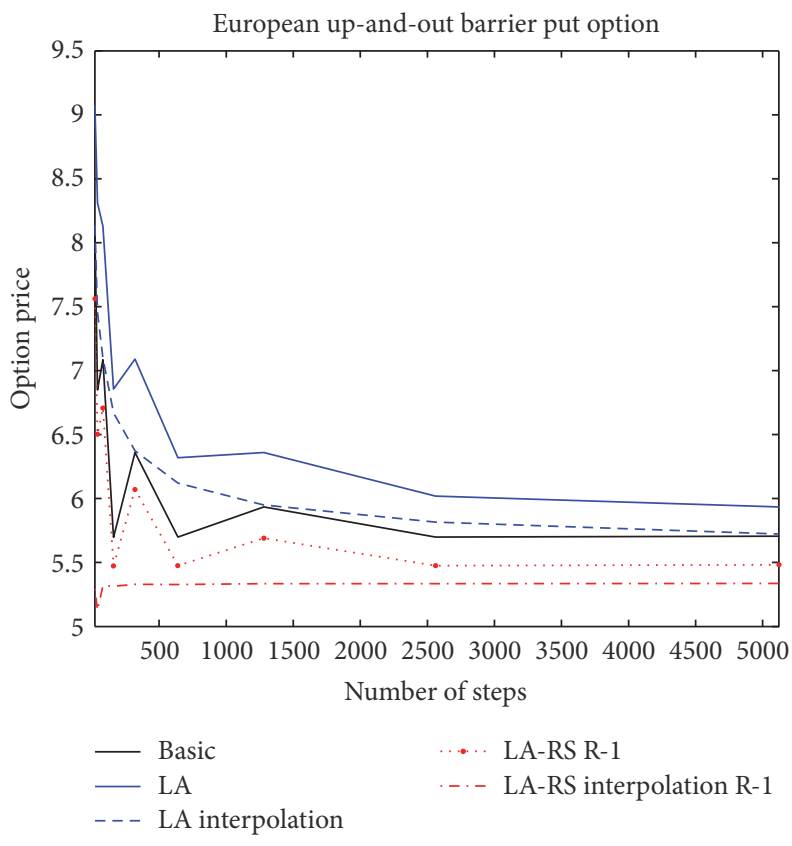

(a) European up-and-out barrier put option, regime 1

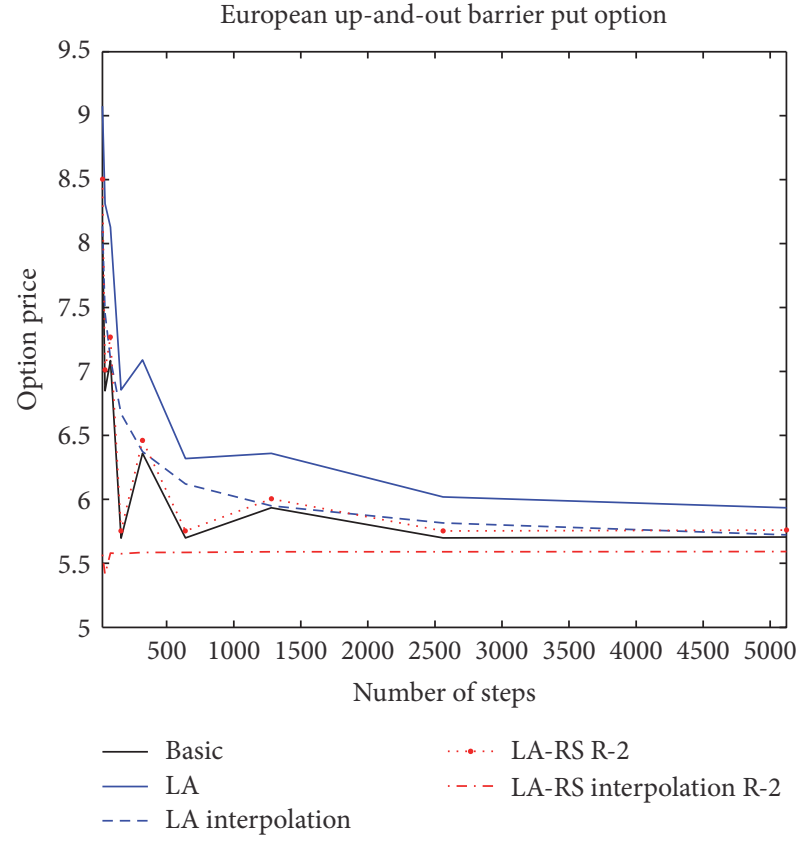

(b) European up-and-out barrier put option, regime 2

FIGURE 4: Convergence of various models for calculation of European up-and-out barrier put option.

$$
\begin{aligned}
& z_{1} \frac{f_{1}-f_{-1}}{2 h}+z_{2} \frac{f_{2}-f_{-2}}{4 h}+z_{3} \frac{f_{3}-f_{-3}}{6 h} \\
& =\left(z_{1}+z_{2}+z_{3}\right) f_{0}^{\prime}+\left(z_{1}+4 z_{2}+9 z_{3}\right) \frac{h^{2}}{3 !} f_{0}^{(3)} \\
& \quad+\cdots .
\end{aligned}
$$

In order to obtain fourth-order accuracy, the following equations must be satisfied:

$$
\begin{aligned}
1+2 y_{1}+2 y_{2} & =z_{1}+z_{2}+z_{3} \\
6 y_{1}+24 y_{2} & =z_{1}+4 z_{2}+9 z_{3} .
\end{aligned}
$$



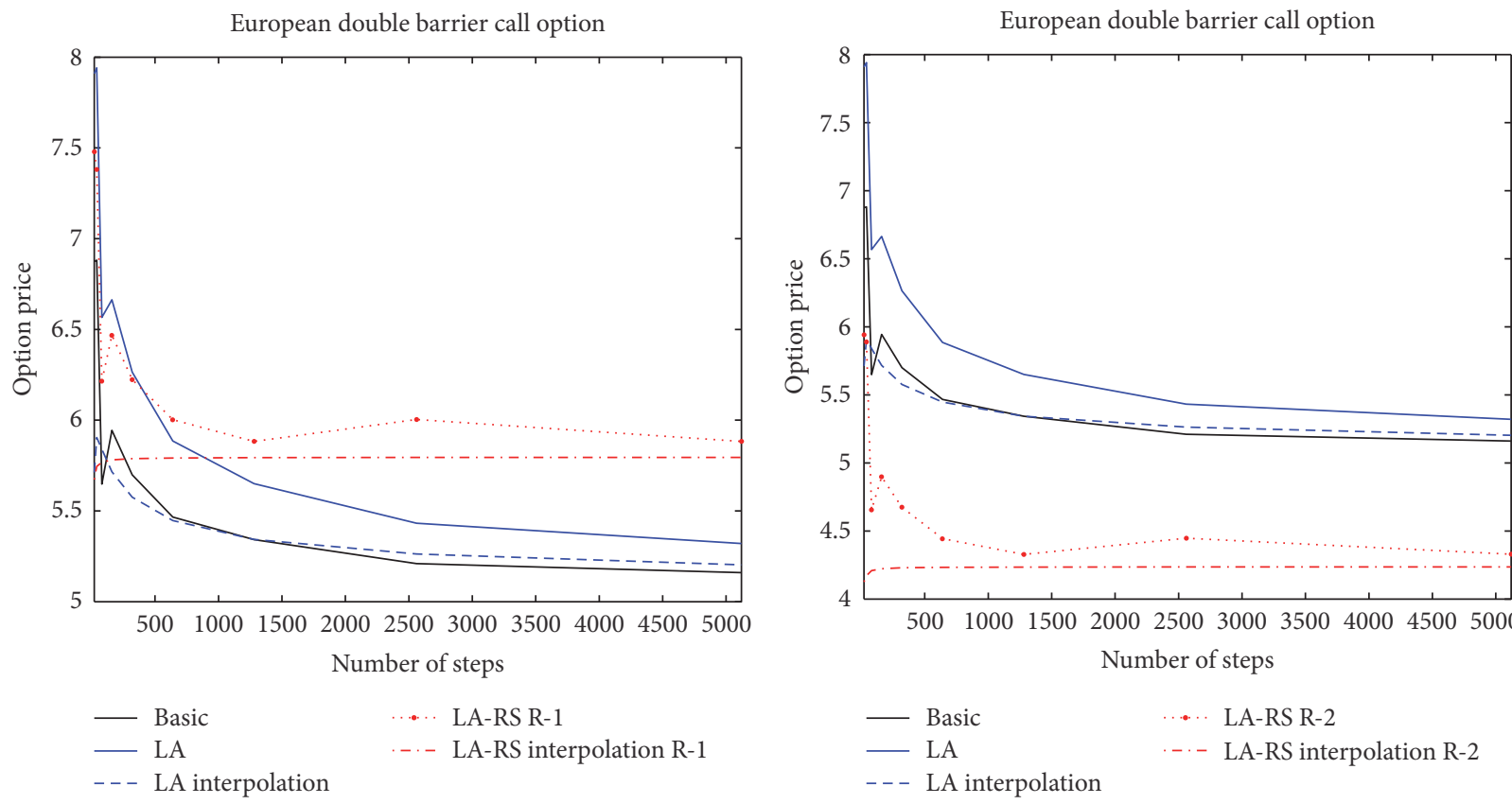

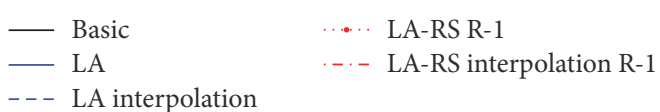

(a) European double barrier call option, regime 1

(b) European double barrier call option, regime 2

FIGURE 5: Convergence of various models for calculation of European double barrier call option.

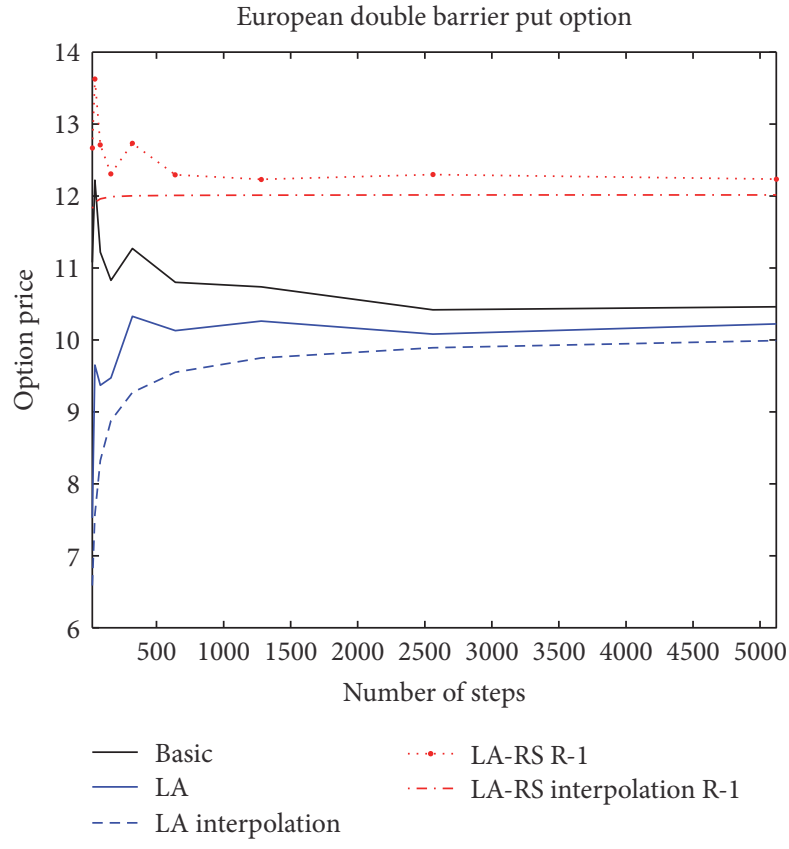

(a) European double barrier put option, regime 1

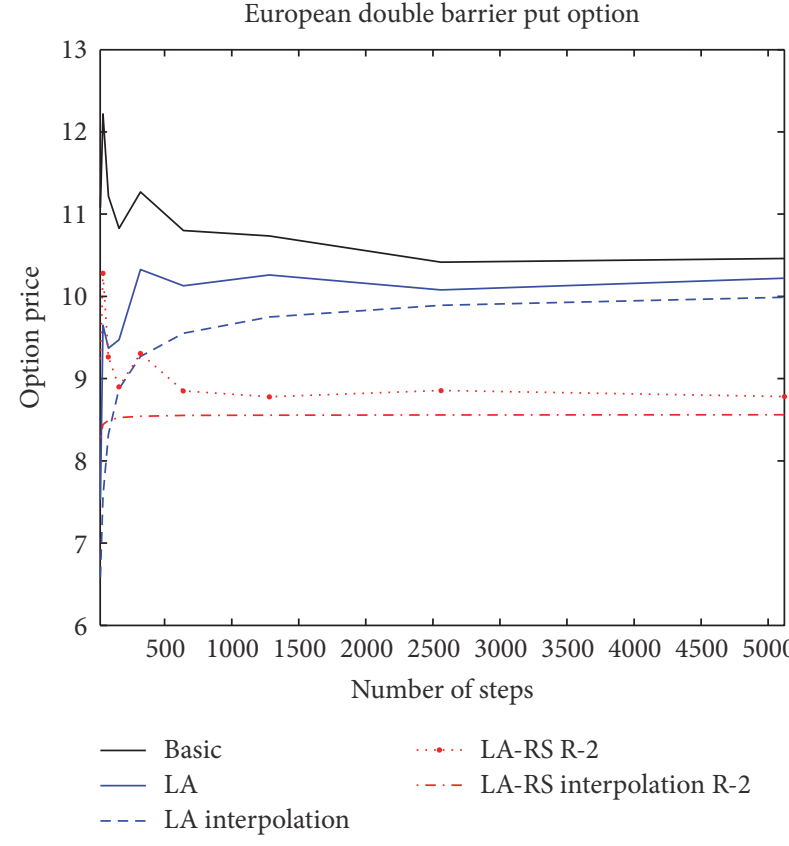

(b) European double barrier put option, regime 2

FIGURE 6: Convergence of various models for calculation of European double barrier put option.

If we take $y_{1}=y_{2}=z_{2}=0, z_{1}=9 / 8$, and $z_{3}=-1 / 8$, we have the following approximation equation of $f_{0}^{\prime}$ :

$$
f_{0}^{\prime}=\frac{9}{8} \frac{f_{1}-f_{-1}}{2 h}-\frac{1}{8} \frac{f_{3}-f_{-3}}{6 h} .
$$

For given regime $i$, let us define the function

$$
f^{i}(x):=\int_{Y(0)-3 h / 2}^{x} V_{0}^{i}(s) d s
$$


We then have

$$
\begin{aligned}
f\left(Y(0)+\frac{h}{2}\right)-f\left(Y(0)-\frac{h}{2}\right) \\
=\int_{Y(0)-h / 2}^{Y(0)+h / 2} V_{0}^{i}(s) d s=h \bar{V}_{0,0}^{i}, \\
f\left(Y(0)+\frac{3 h}{2}\right)-f\left(Y(0)-\frac{3 h}{2}\right) \\
=\int_{Y(0)-3 h / 2}^{Y(0)+3 h / 2} V_{0}^{i}(s) d s \\
=\int_{Y(0)-3 h / 2}^{Y(0)-h / 2} V_{0}^{i}(s) d s+\int_{Y(0)-h / 2}^{Y(0)+h / 2} V_{0}^{i}(s) d s \\
\quad+\int_{Y(0)+h / 2}^{Y(0)+3 h / 2} V_{0}^{i}(s) d s \\
=h\left(\bar{V}_{0,-1}^{i}+\bar{V}_{0,0}^{i}+\bar{V}_{0,1}^{i}\right) .
\end{aligned}
$$

From (A.4) and $\left(f^{i}\right)^{\prime}(Y(0))=V_{0}^{i}(Y(0))$, we finally obtain the following accurate approximation:

$$
\begin{aligned}
V_{0}^{i}(Y(0)) & =\frac{9}{8} \frac{h \bar{V}_{0,0}^{i}}{h}-\frac{1}{8} \frac{h\left(\bar{V}_{0,-1}^{i}+\bar{V}_{0,0}^{i}+\bar{V}_{0,1}^{i}\right)}{3 h} \\
& =-\frac{1}{24} \bar{V}_{0,-1}^{i}+\frac{13}{12} \bar{V}_{0,0}^{i}-\frac{1}{24} \bar{V}_{0,1}^{i} .
\end{aligned}
$$

\section{Competing Interests}

The authors declare that there are no competing interests regarding the publication of this paper.

\section{Acknowledgments}

This research was supported by the National Research Foundation of Korea grant funded by the Korea government (MSIP) (NRF-2015R1C1A1A02037533).

\section{References}

[1] R. C. Merton, "Theory of rational option pricing," The Bell Journal of Economics and Management Science, vol. 4, no. 1, pp. 141-183, 1973.

[2] M. Rubinstein and E. Reiner, "Breaking down the barriers," Risk, vol. 4 , no. 8, pp. 28-35, 1991.

[3] N. Kunitomo and M. Ikeda, "Pricing options with curved boundaries," Mathematical Finance, vol. 2, no. 4, pp. 275-298, 1992.

[4] H. Geman and M. Yor, "Pricing and hedging double-barrier options: a probabilistic approach," Mathematical Finance, vol. 6, no. 4, pp. 365-378, 1996.

[5] J. Sidenius, "Double barrier options: valuation by path counting," The Journal of Computational Finance, vol. 1, no. 3, pp. 6379, 1998.

[6] X. S. Lin, "Double barrier hitting time distributions with applications to exotic options," Insurance: Mathematics and Economics, vol. 23, no. 1, pp. 45-58, 1998.
[7] P. Buchen and O. Konstandatos, "A new approach to pricing double-barrier options with arbitrary payoffs and exponential boundaries," Applied Mathematical Finance, vol. 16, no. 5-6, pp. 497-515, 2009.

[8] J. D. Hamilton, "A new approach to the economic analysis of nonstationary time series and the business cycle," Econometrica, vol. 57, no. 2, pp. 357-384, 1989.

[9] V. Naik, "Option valuation and hedging strategies with jumps in the volatility of asset returns," The Journal of Finance, vol. 48, no. 5, pp. 1969-1984, 1993.

[10] J. Buffington and R. J. Elliott, "American options with regime switching," International Journal of Theoretical and Applied Finance, vol. 5, no. 5, pp. 497-514, 2002.

[11] X. Guo and Q. Zhang, "Closed-form solutions for perpetual American put options with regime switching," SIAM Journal on Applied Mathematics, vol. 64, no. 6, pp. 2034-2049, 2004.

[12] P. P. Boyle and T. Draviam, "Pricing exotic options under regime switching," Insurance: Mathematics and Economics, vol. 40, no. 2, pp. 267-282, 2007.

[13] R. J. Elliott, T. K. Siu, and L. Chan, "On pricing barrier options with regime switching," Journal of Computational and Applied Mathematics, vol. 256, pp. 196-210, 2014.

[14] N. P. B. Bollen, "Valuing options in regime-switching models," The Journal of Derivatives, vol. 6, no. 1, pp. 38-49, 1998.

[15] R. H. Liu and J. L. Zhao, "A lattice method for option pricing with two underlying assets in the regime-switching model," Journal of Computational and Applied Mathematics, vol. 250, pp. 96-106, 2013.

[16] F. L. Yuen and H. Yang, "Option pricing with regime switching by trinomial tree method," Journal of Computational and Applied Mathematics, vol. 233, no. 8, pp. 1821-1833, 2010.

[17] M. Costabile, A. Leccadito, I. Massabó, and E. Russo, "Option pricing under regime-switching jump-diffusion models," Journal of Computational and Applied Mathematics, vol. 256, pp. 152-167, 2014.

[18] M. Costabile, "A lattice-based model to evaluate variable annuities with guaranteed minimum withdrawal benefits under a regime-switching model," Scandinavian Actuarial Journal, 2015.

[19] K.-S. Moon and H. Kim, "An adaptive averaging binomial method for option valuation," Operations Research Letters, vol. 41, no. 5, pp. 511-515, 2013.

[20] J. Kilander, Calibrating an option pricing model under regimeswitching volatility [M.S. thesis], Stockholm School of Economics, Department of Finance, Stockholm, Sweden, 2007.

[21] R. Baule and M. Wilkens, "Lean trees-a general approach for improving performance of lattice models for option pricing," Review of Derivatives Research, vol. 7, no. 1, pp. 53-72, 2004.

[22] E. Omberg, "A note on the convergence of binomial-pricing and compound-option models," The Journal of Finance, vol. 42, no. 2, pp. 463-469, 1987. 


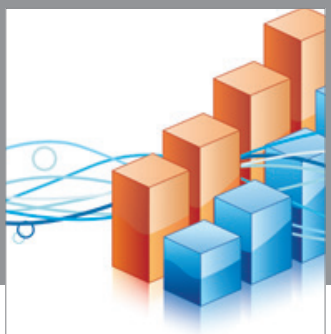

Advances in

Operations Research

vatem alat4

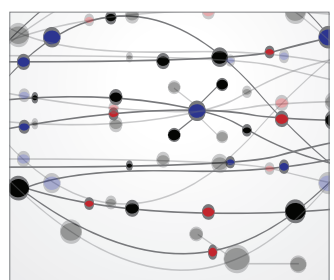

\section{The Scientific} World Journal
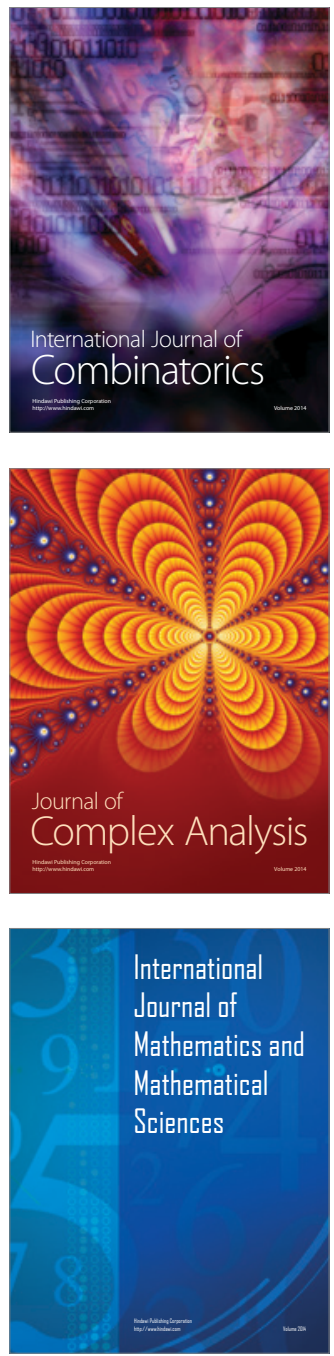
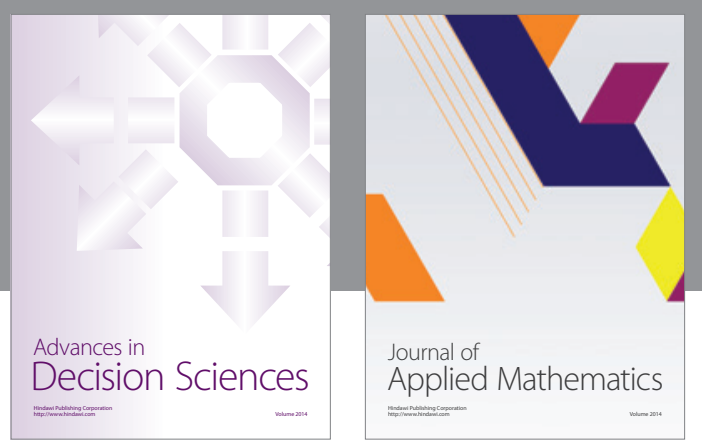

Algebra

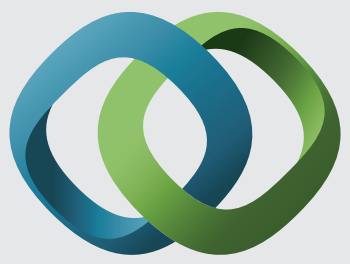

\section{Hindawi}

Submit your manuscripts at

http://www.hindawi.com
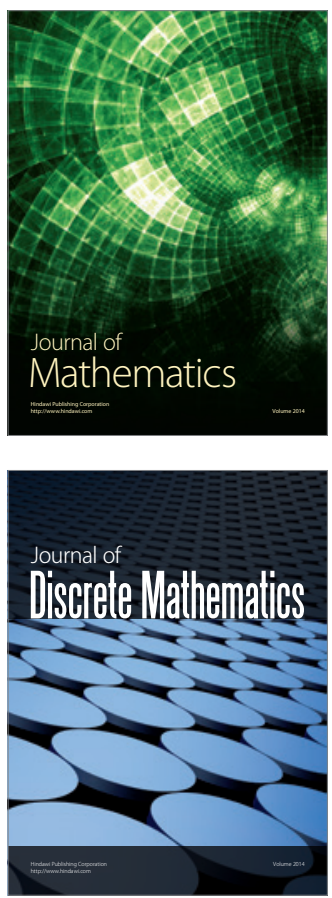

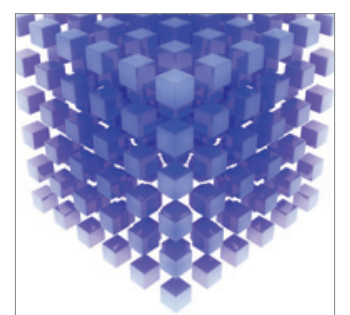

Mathematical Problems in Engineering
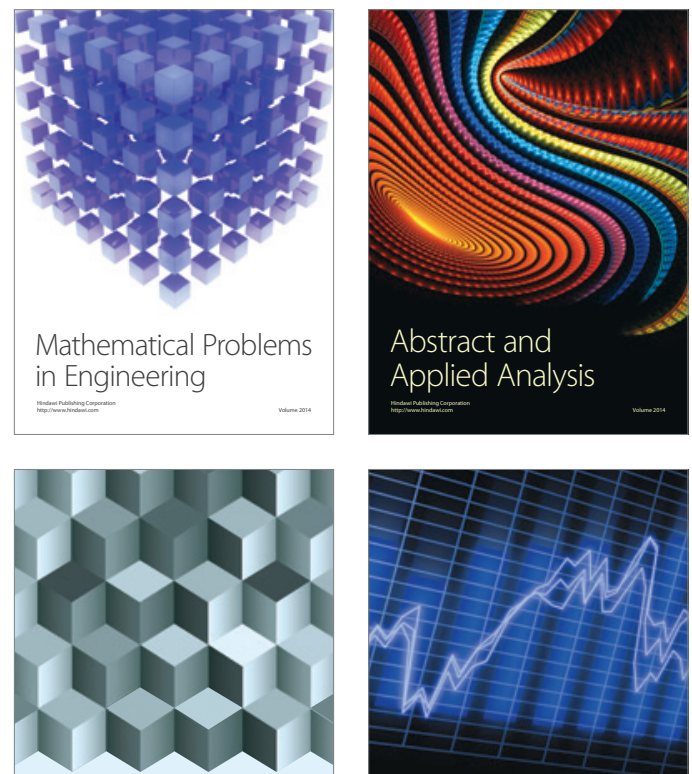

Journal of

Function Spaces

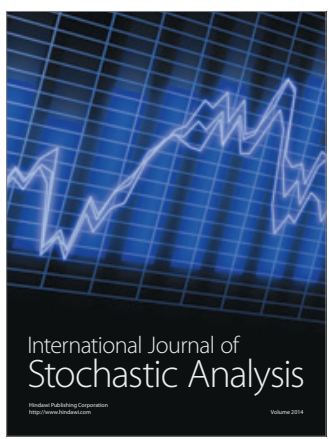

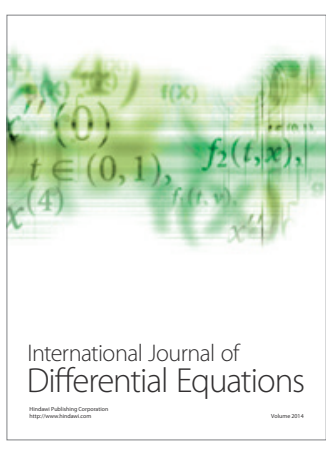
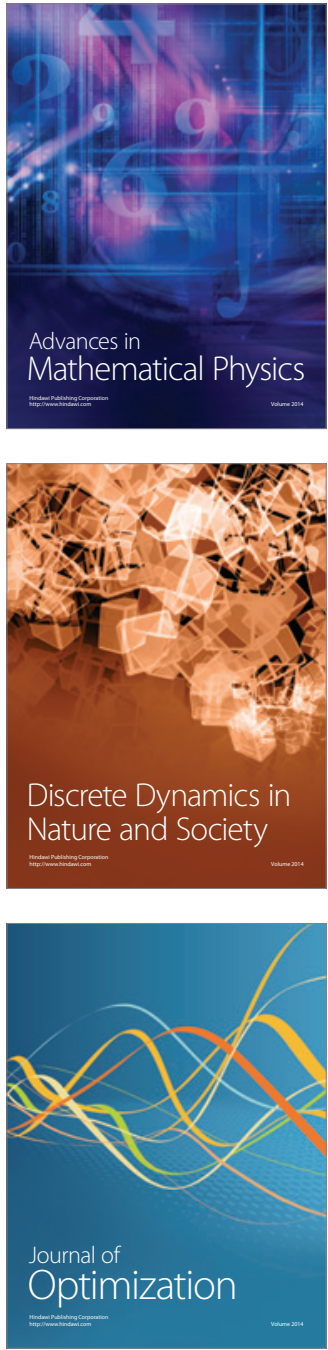